\title{
An Investigation of the Gains from Commitment in Monetary Policy*
}

\author{
Ernst Schaumburg $^{\dagger}$ and Andrea Tambalotti ${ }^{\ddagger}$
}

First Draft: December 2000

This Version: August 2003

\begin{abstract}
This paper proposes a simple framework for analyzing a continuum of monetary policy rules characterized by differing degrees of credibility, in which commitment and discretion become special cases of what we call quasi commitment. The monetary policy authority is assumed to formulate optimal commitment plans, to be tempted to renege on them, and to succumb to this temptation with a constant exogenous probability known to the private sector. By interpreting this probability as a continuous measure of the (lack of) credibility of the monetary policy authority, we investigate the welfare effect of a marginal increase in credibility. Our main finding is that, in a simple model of the monetary transmission mechanism, most of the gains from commitment accrue at relatively low levels of credibility. In our benchmark calibration, a commitment expected to last for only 6 quarters is enough to bridge $75 \%$ of the welfare gap between discretion and commitment. This seems to justify the well known concern of monetary policy makers about their credibility, even in a world with limited access to commitment technologies.
\end{abstract}

JEL Classification: E52, E58, E61

Keywords: Commitment, discretion, credibility, welfare

\footnotetext{
${ }^{*}$ We wish to thank two anonymus referees, Alan Blinder, Pierre-Olivier Gourinchas, Chris Sims and seminar partecipants at Princeton University and the Federal Reserve Bank of New York for useful comments and especially Michael Woodford for several conversations and valuable advice. The views expressed in this paper are those of the authors and do not necessarily reflect the position of the Federal Reserve Bank of New York or the Federal Reserve System.

${ }^{\dagger}$ Kellogg Graduate School of Management, Northwestern University. Email: e-schaumburg@northwestern.edu

${ }^{\ddagger}$ Corresponding Author: Federal Reserve Bank of New York, 33 Libery Street, 3rd Floor; New York, NY 10045.
} Ph: (212) 720-5657. Fax: (212) 720-1844. Email: Andrea.Tambalotti@ny.frb.org 
“... the key point here is that neither of the two modes of central bank behavior rule-like or discretionary - has as yet been firmly established as empirically relevant or theoretically appropriate. (...) This position does not deny that central banks are constantly faced with the temptation to adopt the discretionary policy action for the current period; it just denies that succumbing to this temptation is inevitable."(McCallum, 1999 pg.1489-1490.)

\section{Introduction}

As first pointed out by Kydland and Prescott (1977), "economic planning is not a game against nature but, rather, a game against rational economic agents." When agents are rational and forward looking, economic planners face constraints that depend on their current and future choices, as forecasted by the private sector. Optimal policy therefore needs to internalize the effect of those choices on current private behavior. ${ }^{1}$

Consider for example the case of a central bank confronting a trade-off between inflation and the level of real activity. ${ }^{2}$ In this environment, the credible announcement of a future policy tightening, in excess of that needed to curb forecasted inflationary pressures, lowers inflation expectations, thereby contributing to an easing of the current trade-off. Optimal policy will then exhaust the net marginal benefit of this announcement. On the other hand, following the recursive logic of dynamic programming, discretionary policy will treat private expectations as given, thus foregoing the gains from future announcements. This results in decisions that, although suboptimal, are time-consistent. Optimal policy however, as far as it involves non-verifiable promises about future behavior, is not time-consistent. The contractionary policy that was optimal when the plan was formulated, due to its moderating effect on inflation expectations, ceases to be optimal as soon as those expectations have crystallized. This creates an obvious tension between the optimality of the commitment plan and the ex post incentive to abandon it. Central banks can enjoy the benefits of commitment only if the private sector knows (with probability one) that they will not deviate from their initial plan. But in the absence of a commitment technology, there is no reason for the private sector to believe that this deviation will not happen ex post. This tension is exacerbated by the binary nature of the choice faced by the public. It can either believe the central bank, or not.

In this paper, we propose a way of endowing private agents with the benefit of the doubt. We assume that a new central banker is appointed with a constant and exogenous probability $\alpha$ every period. When a new central banker comes into office (in period $\tau_{j}$ ), she reneges on the promises

\footnotetext{
1 To our knowledge, Fischer (1980) was the first to stress that time inconsistency of the optimal policy is a consequence of forward looking behavior, rather than of rational expectations per se.

${ }^{2}$ After the seminal contributions of Kydland and Prescott (1977) and Calvo (1978), applications of the theory of economic planning with rational agents to monetary policy have attracted by far the most attention in the literature. Following this tradition, we illustrate most of our points within a monetary model. As it will be clear from section 2 though, our theory of policy making under limited commitment has a much wider range of applications.
} 
of her predecessor and commits to a new policy plan that is optimal as of period $\tau_{j}$. Agents understand the possibility and the nature of this change and form expectations accordingly. The private sector will then be constantly doubtful about the reliability of outstanding promises on future policy. In this economy, policymakers have access only to a limited commitment technology, which we refer to as quasi commitment. Individual central bankers can guarantee their own promises but cannot influence the behavior of their successors, who are therefore expected to formulate their own policy plan. Given this expectation, it is optimal for a new central banker to reoptimize.

Note that, even if reoptimizations can be arbitrarily frequent, under quasi commitment there is no room "to exploit temporarily given inflationary expectations for brief output gains." (McCallum, 1999, pg. 1487). Since policy cannot deviate systematically from the behavior anticipated by the private sector, commitment is the global optimum of the planning problem, but it can only be sustained by a technology that guarantees policy announcements with probability one. By placing restrictions on the menu of credible promises available to the planner, quasi commitment then results in suboptimal outcomes. This allows us in turn to rank a continuum of intermediate cases between commitment and discretion and to investigate to what extent access to imperfect commitment technologies might still approximate the optimal equilibrium.

It is worth stressing here that the main source of the central bankers' temptation to abandon the existing policy plan is not the celebrated average inflation bias of Barro and Gordon (1983). As further clarified in section 2, we model a monetary authority whose objective is compatible with the underlying steady state of the economy, and that does not attempt to achieve "overambitious" output gains (Svensson, 2001). Nevertheless, as stressed for example by Clarida et al. (1999) and Woodford (1999), the forward looking nature of agents' expectations makes it optimal for the planner to promise future policies that she would rather abandon ex post. Note that this requires incoming policymakers to contemplate policy from a $\tau_{j}$-optimal perspective. In fact, under this approach to policy, expectations formed in period $\tau_{j}-1$ are taken as given and at $\tau_{j}$ a new policy is formulated that is not necessarily consistent with those expectations. Yet, quasi commitment remains a systematic approach to policy because the public anticipates this to happen with the correct frequency, so that in equilibrium expectations are not exploited on average. Note also that, if at time $\tau_{j}$ policy were reconsidered from a timeless perspective, quasi commitment would collapse to the optimal stationary policy for any value of the parameter $\alpha .^{3}$

Quasi commitment is a useful modeling device to escape the strict binary logic of the debate on "commitment vs. discretion", and to connect those two extreme modes of policy making into a continuum of policy rules. We find it particularly suggestive though to interpret $\alpha$ as a measure of the credibility of the central bank, that is of "the extent to which beliefs about the current and future course of economic policy are consistent with the program originally announced by policymakers," (Blackburn and Christensen, 1989 pg. 2). In an environment in which the available commitment technology suffers the limitations described above, the public always contemplates the possibility that the current policy plan be abandoned. The higher the probability attributed to

\footnotetext{
${ }^{3}$ See Svensson and Woodford (1999) for the concepts of $t_{0}$-optimal and timelessly optimal policy.
} 
this event, the higher the mismatch between the public's perceptions and the program announced by the policymaker, the lower her credibility. ${ }^{4}$ Credibility then becomes a continuous variable, measuring the probability that a central bank matches deeds to words, according to the definition advocated by Blinder (1998).

Our definition of credibility is quite distinct from the ones previously proposed in the literature. In particular, we do not interpret credibility in terms of the relative weight on output in the central bank's loss function (Rogoff, 1985), or in terms of the discrepancy between inflation expectations and the central bank's inflation rate target (Faust and Svensson, 2000), but rather in terms of the expected durability of policy commitments. In this sense then, our notion of credibility is closely related to the empirical measure of actual central bank independence proposed by Cukierman (1992), the observed turnover rate of central bank governors. ${ }^{5}$ Moreover, assuming that private agents are perfectly informed about the nature and objectives of monetary policy, we rule out reputational equilibria based on the public's uncertainty about the preferences of different types of central bankers (see for example Barro, 1986 and Rogoff, 1989).

We also view our approach to credibility as an alternative to the one built on the game theoretic apparatus of Abreu, Pierce and Stacchetti $(1986,1990) .{ }^{6}$ Differently from that literature, we are not interested here in exploring the set of competitive equilibria that can be sustained by punishing governments that renege on their promises. This issue is bypassed by simply assuming that policymakers have access to a commitment technology that guarantees (some of) their promises. In our model, credibility is not the attribute of a particular policy plan - the plan which policymakers optimally choose not to deviate from when behaving sequentially - but rather of a central bank as perceived by the public. In the reputation literature, policy plans are either sustainable or not, mainly as a function of how harsh a punishment the private sector is able to inflict on a deviating policymaker. Optimal policy will therefore never imply a deviation from announced plans (Phelan, 2001). Under quasi commitment on the contrary, different central banks enjoy different levels of credibility, depending on the commitment technology available to them, so that deviations from pre-announced plans are indeed observed in equilibrium, even if they do not generate any systematic surprise for the public. In this sense then, quasi commitment can be thought of as assuming, rather than explaining, policy credibility. Nevertheless, we do not regard this as a major shortcoming of the model, since our focus is on the consequences of marginal increases in credibility, rather than on its premises. As Calvo pricing is widely regarded as a useful starting point to explore the consequences of sticky prices, even if its time dependent microfoundations are only suggestive of actual pricing behavior, so we propose quasi commitment as a modeling device to explore intermediate decision making procedures between discretion and

\footnotetext{
${ }^{4}$ Credibility and $\alpha$, the instantaneous probability of a reoptimization, are inversely related. We find it convenient to adopt $\alpha^{-1}$, the average length of the period between successive reoptimizations, as a direct measure of credibility. We will sometimes refer to the period between successive reoptimizations as "a regime".

${ }^{5}$ Cukierman, Webb and Neyapti (1992) show that central bankers' turnover rates are correlated with the level and variability of inflation in cross country regressions.

${ }^{6}$ The seminal papers are Chari and Kehoe (1990) and Stokey (1989, 1991). See Ljungqvist and Sargent (2000, Chapter 16) for an introduction and Phelan and Stacchetti (2001) for a prominent recent example.
} 
commitment, along a dimension that can be usefully interpreted as reflecting differing degrees of credibility.

The papers in the literature that are closest to ours are Flood and Isard (1988) and Roberds (1987). ${ }^{7}$ Flood and Isard (1988) identify conditions under which commitment to a simple rule can be improved upon by the addition of a provision for discretionary optimization in the face of "big" shocks. ${ }^{8}$ Our framework can easily be adapted to interpret $\alpha$ as the probability of an extreme draw of the exogenous i.i.d. shocks that buffet the economy. Under this interpretation, policymakers are given free reign in the face of "big" shocks, but differently from Flood and Isard (1988), this optimally results in a new commitment rather than in a reversion to discretion.

Roberds (1987) presents a technical apparatus very similar to the one described below. She solves a model in which policy is set by a sequence of policymaking administrations whose turnover is determined by i.i.d. Bernoulli draws. Thanks in part to the better understanding of optimal policy problems available today, our solution method is more transparent and more widely applicable than Roberds', accommodating systems that include non-trivial dynamics for the endogenous state variables and singularities in the contemporaneous relations. Moreover, we prove the applicability of standard linear quadratic techniques to the stochastic replanning problem, which was only assumed to hold in Roberds (1987). We also provide analytical expressions for the value of each administration's problem as a function of the parameter $\alpha$, and for the impulse response functions of the endogenous variables to the exogenous shocks. On the other hand, we do not treat here the case of asymmetric information between policymakers and the public, that gives rise to an interesting class of equilibria with delayed information (see Roberds, 1987 Section 4). Finally, and much more importantly in our view, we provide a novel interpretation of the frequency of administration turnover as a measure of the credibility of policy in the eyes of the public. This in turn allows us to exploit the technical apparatus first presented by Roberds (1987), and further developed here, to address important questions in the still open debate on "rules vs. discretion."

The remainder of the paper is organized as follows. Section 2 formally introduces the notion of quasi commitment and outlines our proposed solution method for finding quasi commitment equilibria. The discussion is cast in terms of a general linear-quadratic economy with rational, forward looking expectations, with most technical details relegated to an appendix. In Section 3 this methodology is applied to a simple New Keynesian model of the monetary transmission mechanism. We first solve the model analytically, to gain some intuition into the key steps involved in finding a quasi commitment equilibrium. We then proceed to illustrate the dynamic behavior of the economy in a calibrated version of the model, under different assumptions on the degree of credibility enjoyed by its monetary authority. Finally, we study the distribution of the gains from commitment and the extent to which a limited commitment technology can still approximate the

\footnotetext{
${ }^{7}$ Fischer (1980, pg. 105) could be interpreted as foreshadowing quasi commitment. However, his conjecture that "a randomized policy that is rationally expected may do better than non-stochastic optimal open loop policy" (i.e. full commitment) does not hold true in our framework. Kara (2002) proposes an empirical study of quasi commitment.

${ }^{8}$ Flood and Isard (1988) consider a commitment to a simple non contingent rule, which, even if credible, does not provide any insulation against shocks. That is why adding an escape clause can improve its performance.
} 
optimal outcome. Section 4 concludes.

\section{An Analysis of Quasi Commitment}

This section describes a general analytical framework for the study of a continuum of policy rules indexed by a credibility parameter $\alpha \in[0 ; 1] .^{9}$ Two common assumptions about policymakers' credibility, discretion (no credibility) and commitment (perfect credibility), are nested in this framework as limiting cases of what we call quasi commitment. Several popular models of policy making with forward looking agents fall into the class of models treated here, including for example models of monetary policy and taxation. While much of the discussion in the remainder of the paper focuses on the leading example of monetary policy, this section avoids reference to any specific economic context in order to highlight the general nature of the approach.

\subsection{The Economic Environment}

Consider a situation in which a sequence of policymakers with tenures of random duration seek to maximize a shared policy objective. Each policymaker can commit to a contingent plan for the duration of her tenure, but cannot make credible promises regarding the actions of her successors. At the beginning of every period the acting policymaker receives a publicly observable signal $\eta_{t} \in\{0,1\}$. If $\left\{\eta_{t}=1\right\}$, the incumbent is replaced at the beginning of period $t$, an event which occurs with some fixed probability $\alpha$ known to all agents in the economy. To make things simple, the sequence of regime change signals $\left\{\eta_{t}\right\}_{t \geq 0}$ is assumed to be i.i.d. over time and exogenous with respect to all other randomness in the system. ${ }^{10}$ When a regime change is signalled (i.e. $\left.\eta_{t}=1\right)$, a new policymaker takes over and formulates a policy plan, effective as of time $t$. If no regime change is signalled (i.e. $\eta_{t}=0$ ) the existing policymaker continues to implement the policy she had committed to at the beginning of her tenure.

The state of the economy is described by a set of predetermined state variables $x_{t}$ buffeted by an i.i.d. exogenous shock process $\varepsilon_{t}$. Based on the information set $\mathcal{I}_{t}=\left\{x_{s}, \eta_{s}\right\}_{s \leq t}$ and their knowledge of the parameters of the model, including $\alpha$, private agents form expectations about the future resulting in allocations described by the jump variables $\left\{X_{t}\right\}_{t \geq 0}$, while the policymaker implements a sequence of policy actions $\left\{i_{t}\right\}_{t \geq 0}$ according to a state contingent plan committed to at the beginning of her tenure. Rational private agents correctly take into account the probability of a regime change when forming expectations about the future, which therefore depend on the distribution of both types of shocks, $\left\{\varepsilon_{t}, \eta_{t}\right\}_{t \geq 0}$.

The starting point of the analysis is a system of linearized equilibrium conditions from a dynamic stochastic general equilibrium model. The system consists of the deviations from a

\footnotetext{
${ }^{9}$ Following Svensson (1999a), we interpret a policy rule in a broad sense, as a "prescribed guide for (...) policy conduct."

10 The case where the likelihood of regime changes is state-dependent is in general much more complicated. One notable exception, which can easily be incorporated in our framework, is to let $\left\{\eta_{t}\right\}$ depend on the realization of the i.i.d. shocks hitting the system. In this case, reoptimizations could be interpreted as reactions to "big" shocks, as in Flood and Isard (1988).
} 
deterministic steady state of $n_{x}$ predetermined variables $x_{t}$ and $n_{X}$ non-predetermined variables $X_{t}$, whose joint evolution is described by

$$
\left(\begin{array}{c}
x_{t+1} \\
G E_{t} X_{t+1}
\end{array}\right)=A\left(\begin{array}{c}
x_{t} \\
X_{t}
\end{array}\right)+B i_{t}+\left(\begin{array}{c}
\varepsilon_{t+1} \\
0
\end{array}\right), \quad x_{0}=x
$$

for a given sequence $\left\{i_{t}\right\}_{t \geq 0}$, where $i_{t}$ is a $q$-dimensional vector of policy instruments. As usual, autocorrelated shocks are included in $x_{t}$, so that the structural shocks $\left\{\varepsilon_{t}\right\}_{t \geq 0}$ can be assumed to be i.i.d. with covariance matrix $\Omega$. The matrices $\Omega \in \mathbb{R}^{n_{x} \times n_{x}}, A \in \mathbb{R}^{\left(n_{x}+n_{X}\right) \times\left(n_{x}+n_{X}\right)}, G \in \mathbb{R}^{n_{X} \times n_{X}}$ and $B \in \mathbb{R}^{\left(n_{x}+n_{X}\right) \times q}$ contain structural parameters specific to the steady state, which are assumed to be known to all agents.

The policymaker minimizes a discounted sum of expected period losses of the form

$$
L_{t}=\left(\begin{array}{c}
x_{t} \\
X_{t} \\
i_{t}
\end{array}\right)^{\prime} W\left(\begin{array}{c}
x_{t} \\
X_{t} \\
i_{t}
\end{array}\right)
$$

for some positive semidefinite symmetric matrix $W$. Even though it is not always possible to derive (2) from first principles as a welfare criterion, in such cases we will simply assume that the policymaker wishes to stabilize the economy around its structural steady state. In doing so we conform to the established tradition of adopting quadratic objective functions in policy analysis, which allows us to compare our results to those found in the literature.

An important feature of the loss function in (2) is that the policymaker has not been endowed with target levels for the endogenous variables different from their respective steady states, thus eliminating the average bias of the discretionary policy. The reason is that we wish to isolate the effect of forward looking expectations on the conduct of optimal policy and in particular on the gains from commitment. The lack of target values also implies that the steady state and the unconditional expectation of the state variables coincide and remain unchanged across all levels of credibility, which allows us to gauge the relative ability of policymakers to counteract shocks to the economy as a function of their credibility. ${ }^{11}$

The optimal equilibrium that we wish to solve for in the limited commitment setting described above can be summarized by the following

Definition 1 (Quasi Commitment Equilibrium) A Quasi-Commitment Equilibrium with credibility parameter $\alpha$ consists of a sequence $\left\{x_{t}, X_{t}, i_{t}\right\}_{t \geq 0}$, such that

i) Agents one-step-ahead expectations $E_{t}\left[X_{t+1}\right]$ are linear in a suitably expanded set of state variables, which includes $x_{t}$

ii) The policy plan $\left\{i_{t}\right\}_{t \geq 0}$ maximizes (2), given (1) and agents' expectations

\footnotetext{
11 From a purely analytical perspective, target values can be accomodated by simply augmenting (1) with an equation for the constant. Under quasi commitment though, the presence of target values would imply different average levels of inflation at different levels of $\alpha$, since lower credibility implies a higher average bias.
} 
iii) The sequence $\left\{x_{t}, X_{t}\right\}_{t \geq 0}$ is the unique stable solution of (1), given the optimal policy plan $\left\{i_{t}\right\}_{t \geq 0}$ and agents' expectations

iv) Agents' expectations are rational, given the equilibrium sequence $\left\{i_{t}, x_{t}, X_{t}\right\}_{t \geq 0}$ and the probability of a reoptimization $\alpha$

Note that there may well exist equilibria in which rational expectations cannot be represented as linear functions of the state variables. We disregard any such equilibria here, since this would bring us beyond the linear quadratic regulator framework with which we work in the sequel.

\subsection{The Policymaker's Problem}

The problem faced by the policy authority is to minimize the discounted sum of expected period losses (2), subject to the constraints given by the equilibrium conditions (1). The optimization problem can then be written as

$$
\begin{aligned}
L(x)= & \min _{\left\{i_{t}\right\}_{t \geq 0}} E_{0} \sum_{t=0}^{\infty} L_{t} \\
& \text { s.t. } \quad x_{t+1}-A_{11} x_{t}-A_{12} X_{t}-B_{1} i_{t}-\varepsilon_{t+1}=0, \quad x_{0}=x \\
& 1_{\left\{\eta_{t+1}=0\right\}}\left[G E_{t} X_{t+1}-A_{21} x_{t}-A_{22} X_{t}-B_{2} i_{t}\right]=0
\end{aligned}
$$

where the matrices $A, B$ have been partitioned conformably with $\left(x_{t}^{\prime}, X_{t}^{\prime}\right)^{\prime}$. This looks much like the standard commitment problem (see for example Söderlind, 1999), except for the fact that the last set of constraints does not bind whenever a regime change $\left\{\eta_{t+1}=1\right\}$ occurs. This essentially allows a new policymaker to disregard expectations held in the period prior to her arrival into office. As the private sector forms its expectations prior to the realization of the $\eta_{t+1}$ shock, the policymaker is in effect able to "surprise" agents. The term surprise is however a slight misnomer, since agents attach positive probability to a regime change as well as to the continuation of the current regime. In this sense they are equally "surprised" when the regime actually continues for another period.

For the purpose of characterizing its solution, it is useful to analyze the optimal policy problem by grouping the losses accruing during each successive regime, rather than period by period. To this end, define the dates of regime changes $\left\{\tau_{j}\right\}_{j \geq 0}$ and regime durations beyond the initial period $\left\{\Delta \tau_{j}\right\}_{j \geq 0}$ as

$$
\begin{aligned}
& \tau_{j}=\min \left\{t \mid t>\tau_{j-1}, \eta_{t}=1\right\}, \tau_{0} \equiv 0 \\
& \Delta \tau_{j}=\tau_{j+1}-\tau_{j}-1
\end{aligned}
$$

Thus the $j^{\text {th }}$ regime starts at date $t=\tau_{j}$ and is in effect for $t \in\left\{\tau_{j}, \ldots, \tau_{j}+\Delta \tau_{j}\right\}$, that is until time $t=\tau_{j+1}$, at which point the $j+1^{\text {st }}$ regime takes effect. With this notation the policymaker's objective (3) can be rewritten in terms of a sum of losses over individual policy regimes

$$
\begin{aligned}
L(x)=\min _{\left\{i_{t}\right\}_{t \geq 0}} E_{0} & \sum_{j=0}^{\infty} \beta^{\tau_{j}}\left[\sum_{k=0}^{\Delta \tau_{j}} L_{\tau_{j}+k}\right] \\
\text { s.t. } \quad & x_{t+1}-A_{11} x_{t}-A_{12} X_{t}-B_{1} i_{t}-\varepsilon_{t+1}=0, \quad x_{0}=x \\
& 1_{\left\{\eta_{t+1}=0\right\}}\left(G E_{t} X_{t+1}-A_{21} x_{t}-A_{22} X_{t}-B_{2} i_{t}\right)=0
\end{aligned}
$$


The recursive structure of the problem is now clear: policymakers face exactly the same type of problem at the beginning of each regime. In the initial period the expectational constraint is not binding, although it must be satisfied in every period thereafter. Furthermore, since the sequence of regime durations $\left\{\Delta \tau_{j}\right\}_{j \geq 0}$ is i.i.d. Geometric $(\alpha)$, the expected duration of each regime is equal to $\alpha^{-1}$.

The solution to a problem of this type can be found using a version of the Bellman optimality principle, which implies that successive central bankers choose to implement the same optimal plan. Problem (5) can then be interpreted as that of a single policymaker who cares not only about the losses accruing during her own regime, but also takes into account a terminal payoff given by the discounted sum of losses pertaining to all subsequent regimes. The associated Bellman equation is then ${ }^{12}$

$$
\begin{aligned}
& V\left(x_{\tau_{j}}\right)= \min _{\left\{x_{k+1}, X_{k}, i_{k}\right\}_{k=\tau_{j}}^{\infty}} \max _{\left\{\varphi_{k+1}\right\}_{k=\tau_{j}}^{\infty}} E_{\tau_{j}}\left\{\sum _ { k = 0 } ^ { \Delta \tau _ { j } } \beta ^ { k } \left[L_{\tau_{j}+k}+\beta^{\Delta \tau_{j}+1} V\left(x_{\tau_{j+1}}\right)\right.\right. \\
&\left.\left.+2 \varphi_{\tau_{j}+k+1}^{\prime}\left(G E_{\tau_{j}+k} X_{\tau_{j}+k+1}-A_{21} x_{\tau_{j}+k}-A_{22} X_{\tau_{j}+k}-B_{2} i_{\tau_{j}+k}\right)\right]\right\} \\
& \text { s.t. } \quad x_{\tau_{j}+k+1}-A_{11} x_{\tau_{j}+k}-A_{12} X_{\tau_{j}+k}-B_{1} i_{\tau_{j}+k}-\varepsilon_{\tau_{j}+k+1}=0 \\
& \quad \varphi_{\tau_{j}}=0
\end{aligned}
$$

In this expression, the state variables $x_{\tau_{j}}$ are predetermined as of the last period of the $j-1^{\text {st }}$ regime while the $n_{X}$ predetermined Lagrange multipliers $\varphi_{\tau_{j}+k+1}$, corresponding to the constraints involving private agent's expectations, must satisfy the initial condition $\varphi_{\tau_{j}}=0$. This is a consequence of the reoptimization that accompanies the inception of each regime, whereby the constraint involving expectations formed in the last period of the previous regime is not binding for the incoming policymaker. Therefore, the value function $V(\cdot)$ depends on $x_{\tau_{j}}$ alone, rather than on the predetermined Lagrange multipliers as well. Note that the "regime-by-regime" formulation in (6) requires us to evaluate the value function only at points in time $\left\{\tau_{j}\right\}_{j \geq 0}$ when a new regime begins, instead of period-by-period, when the value function depends explicitly on the evolution of the Lagrange multipliers. In accordance with the Bellman principle, the value function $V(x)$ defined in (6) is the minimum achievable value of the objective (2) and the state contingent optimal policy plan chosen by the $j^{\text {th }}$ policymaker will indeed be optimal for all subsequent policymakers to follow.

The solution method is very similar to solving for the optimal discretionary (i.e. Markov perfect) equilibrium, since each policymaker reoptimizes without taking into account past commitments. There are, however, a few notable differences. First, the policymaker is in fact able to credibly commit to a policy rule, albeit only for a random number of periods. This results in a running cost function that is random as of time $\tau_{j}$. Second, the $j^{\text {th }}$ central banker must look infinitely into the future because her commitment will last an arbitrarily large number of periods with a positive probability. Through the continuation value, she also internalizes the effect on her successors of the level of the state variables at the end of her tenure. Finally, within the $j^{\text {th }}$

\footnotetext{
${ }^{12}$ See Marcet and Marimon (1999) for a formal treatment of "recursive saddle point" functional equations.
} 
regime, the state variables consist of the $n_{x}$ predetermined variables $\left\{x_{\tau_{j}+k}\right\}_{0 \leq k \leq \Delta \tau_{j}}$ together with the $n_{X}$ Lagrange multipliers $\left\{\varphi_{\tau_{j}+k}\right\}_{0 \leq k \leq \Delta \tau_{j}}$, rather than of the predetermined variables alone, as it would be the case under discretion.

\subsection{Private Agents' Expectations and Linear Equilibria}

In our environment, private agents must form expectations about the entire infinite future, and in particular across policy regimes. Therefore they differ significantly from policymakers, in that they must form expectations about the responses of the non-predetermined variables $X_{t+1}$ to the regime change signal $\eta_{t} \in\{0,1\}$, taking into account that every period, with probability $\alpha$, a regime change may occur. Since we restrict attention to equilibria with linear dynamics, we consider a representation of private agents' rational expectations as linear functions of the state variables within each regime. Due to the exogeneity of the signals $\eta$, agents' expectations are a simple weighted average of expectations conditional on the current regime continuing for another period and of expectations conditional on a regime change. Note that, without the assumed exogeneity of the regime change signals, agents would have to internalize the effect of their own expectation formation on the likelihood of a regime change, which would introduce a significant complication in the analysis.

The one-period-ahead expectations formed by agents in period $k$ of the $j^{\text {th }}$ regime can then be written as

$E_{\tau_{j+k}}\left[X_{\tau_{j}+k+1}\right]=(1-\alpha) E_{\tau_{j+k}}[X_{\tau_{j}+k+1} \mid \underbrace{\tau_{j+1}>\tau_{j}+k+1}_{\text {within regime }}]+\alpha E_{\tau_{j}+k}[X_{\tau_{j}+k+1} \mid \underbrace{\tau_{j+1}=\tau_{j}+k+1}_{\text {across regime }}]$

using Bayes rule to condition on whether a regime change happens. The first expectation is simply the one-step-ahead expectation of $X_{\tau_{j}+k+1}$, given no regime change between today and tomorrow. The second term is an expectation across regimes, which is assumed to be linear in the state variables

$$
\begin{aligned}
& E_{\tau_{j}+k}\left[X_{\tau_{j}+k+1} \mid \tau_{j+1}=\tau_{j}+k+1\right] \\
= & H E_{\tau_{j}+k}\left[x_{\tau_{j}+k+1} \mid \tau_{j+1}=\tau_{j}+k+1\right]+\widetilde{H} E_{\tau_{j}+k}\left[\varphi_{\tau_{j}+k+1} \mid \tau_{j+1}=\tau_{j}+k+1\right] \\
= & H E_{\tau_{j}+k}\left[x_{\tau_{j}+k+1}\right]
\end{aligned}
$$

for some $H \in \mathbb{R}^{n_{X} \times n_{x}}$ and $\tilde{H} \in \mathbb{R}^{n_{X} \times n_{X}}$. The last equality follows from the fact that, conditional on $\left\{\eta_{\tau_{j}+k+1}=1\right\}, \varphi_{\tau_{j}+k+1}=\varphi_{\tau_{j+1}}=0$ because of the intervening reoptimization. Furthermore, the fact that $x_{\tau_{j}+k+1}$ is predetermined implies that knowledge of $\eta_{\tau_{j}+k+1}$ does not help to predict its value, so that the last line in (7) is just conditioned on the information $\mathcal{I}_{\tau_{j}+k}$.

\subsection{Quasi Commitment Equilibrium}

To solve for a quasi commitment equilibrium one must simultaneously solve for the value function $V(\cdot)$, the optimal central bank plan and agents' rational expectations, which in turn feed back into 
the central bank's optimization problem through the matrix $H$ in (7). This suggests an iterative procedure in which one alternates between solving for the value function and the optimal policy rule given private agents' expectations and solving for the expectation representation $H$ consistent with a given policy rule.

Given the assumed linear-quadratic structure of the problem, we start by guessing a quadratic form for the value function, which is parametrized as follows

$$
V(x)=x^{\prime} P x+\rho, \quad P \in \mathbb{R}^{n_{x} \times n_{x}}, \rho \geq 0
$$

The validity of (8) must then be ex post verified as part of the solution. For given beliefs $H$, the Lagrangian corresponding to problem (6) is

$$
\begin{aligned}
\mathcal{L}= & E_{\tau_{j}}\left\{\sum _ { k = 0 } ^ { \infty } ( \beta ( 1 - \alpha ) ) ^ { k } \left[L_{\tau_{j}+k}+\alpha \beta\left(x_{\tau_{j}+k+1}^{\prime} P x_{\tau_{j}+k+1}+\rho\right)\right.\right. \\
& +2 \varphi_{\tau_{j}+k+1}^{\prime}\left((1-\alpha) G E_{\tau_{j}+k}\left[X_{\tau_{j}+k+1} \mid \tau_{j+1}>\tau_{j}+k+1\right]\right. \\
& \left.+\alpha G H x_{\tau_{j}+k+1}-A_{21} x_{\tau_{j}+k}-A_{22} X_{\tau_{j}+k}-B_{2} i_{\tau_{j}+k}\right) \\
& \left.\left.+2 \phi_{\tau_{j}+k+1}^{\prime}\left(x_{\tau_{j}+1+k}-A_{11} x_{\tau_{j}+k}-A_{12} X_{\tau_{j}+k}-B_{1} i_{\tau_{j}+k}\right)\right]\right\}
\end{aligned}
$$

where we have introduced the $n_{x}$ non-predetermined Lagrange multipliers $\phi_{\tau_{j}+k}$. Note that the probability of a regime change has three effects. First, it modifies the discount rate to take into account the survival probability of the regime (the probability of the regime lasting at least $k+1$ periods is $\left.(1-\alpha)^{k}\right)$. Similarly, the continuation value is multiplied by $\alpha(1-\alpha)^{k}$ to account for the probability of the regime ending at time $\tau_{j}+k+1$. Finally, the expectational constraint is modified to take into account that the non-predetermined variables may jump unexpectedly if a regime change should occur at time $\tau_{j}+k+1$.

To see the connection between quasi commitment, discretion and full commitment, consider first $\alpha=1$. In this case only the first term in the infinite sum remains. Since a new regime starts every period with probability one, we have $\forall t, \varphi_{t}=0$ and (9) reduces to the familiar expression for the optimal discretionary policy (see Söderlind, 1999). Next, consider the opposite extreme, $\alpha=0$. In this case, the running cost function is identical to the objective function under commitment, the terminal value drops out of the sum and the term $\alpha G H x_{\tau_{j}+k+1}$ disappears from the constraint.

It is not possible to solve simultaneously for the value function $V$, private agents expectations $H$, and the optimal policy plan. However, it is not difficult to solve for any one of these quantities given the other two. This motivates the following two-step iterative solution procedure.

Step 1 For given values of $H$ and of the value function parameters $(P, \rho)$, the policymaker's problem can be solved by taking first order conditions in (9). The resulting dynamic system yields the optimal within-regime evolution of the extended vector of variables $\left\{x_{t}, \varphi_{t}, i_{t}, X_{t}\right\}$, which we 
write in state space form as $\forall t \in\left\{\tau_{j}, \ldots, \tau_{j}+\Delta \tau_{j}\right\}$

$$
\begin{aligned}
& \left(\begin{array}{l}
x_{t+1} \\
\varphi_{t+1}
\end{array}\right)=M\left(\begin{array}{l}
x_{t} \\
\varphi_{t}
\end{array}\right)+\left(\begin{array}{c}
\varepsilon_{t+1} \\
0
\end{array}\right) \\
& \left(\begin{array}{c}
i_{t} \\
X_{t}
\end{array}\right)=\left(\begin{array}{l}
\xi^{\prime} \\
C
\end{array}\right)\left(\begin{array}{l}
x_{t} \\
\varphi_{t}
\end{array}\right)
\end{aligned}
$$

with $\varphi_{\tau_{j}}=0$ and $x_{\tau_{j}}$ predetermined as of the last period in the $j-1^{\text {st }}$ regime. The matrices $(M, C, \xi)$ are in general complicated non-linear functions of agents' beliefs $H$ and of the value function parameters $(P, \rho)$.

Step 2 Given the state space form (10) one can substitute back into (7) to get an equation for $H$ which must be satisfied in order for expectations to be consistent with the proposed equilibrium

$$
\begin{aligned}
E_{\tau_{j}+k}\left[X_{\tau_{j}+k+1} \mid \tau_{j+1}=\tau_{j}+k+1\right] & =C\left(\begin{array}{l}
I \\
0
\end{array}\right) E_{\tau_{j}+k} x_{\tau_{j}+k+1} \\
& \Rightarrow H=C\left(\begin{array}{c}
I_{n_{x} \times n_{x}} \\
0_{n_{X} \times n_{x}}
\end{array}\right)
\end{aligned}
$$

Finally, one may solve for the value function parameters $(P, \rho)$ by substituting the state space representation (10) into the Bellman equation (6) to obtain

$$
\begin{aligned}
P & =\left(\begin{array}{l}
I \\
0
\end{array}\right)^{\prime} V_{1}\left(\begin{array}{l}
I \\
0
\end{array}\right)+\alpha \beta\left(\begin{array}{l}
I \\
0
\end{array}\right)^{\prime} M^{\prime} V_{2} M\left(\begin{array}{l}
I \\
0
\end{array}\right) \\
\rho & =\frac{\beta}{1-\beta}\left((1-\alpha) \operatorname{tr}\left\{\left(\begin{array}{l}
I \\
0
\end{array}\right)^{\prime} V_{1}\left(\begin{array}{l}
I \\
0
\end{array}\right) \Omega\right\}\right)+\alpha \operatorname{tr}\left\{\left(\begin{array}{l}
I \\
0
\end{array}\right)^{\prime} V_{2}\left(\begin{array}{l}
I \\
0
\end{array}\right) \Omega\right\}
\end{aligned}
$$

where the matrices $V_{1}, V_{2}$ are given in the appendix as solutions to two Sylvester equations involving the state space parameters $\{M, C, \xi\}$ alone.

Starting with some initial guess for $(H, P, \rho)$, a fixed point for this procedure will result in a state contingent policy plan $i_{t}=\xi^{\prime}\left(\begin{array}{l}x_{t} \\ \varphi_{t}\end{array}\right)$ which is optimal from the perspective of the policymaker and in private expectations which are rational given the nature of the policymaker's limited commitment.

\section{Quasi Commitment and Monetary Policy}

This section applies the methods developed above to a simple New Keynesian model of the monetary transmission mechanism. ${ }^{13}$ The model describes an economy populated by a continuum of competitive, optimizing private agents and by a central bank that tries to maximize (a second order approximation to) the welfare of the representative consumer. The strategic interaction

\footnotetext{
13 Woodford (2002) contains an exhaustive treatment of this class of models. See also Clarida et al. (1999) and Galí (2001).
} 
between the atomistic private sector and the monetary authority leads to the joint determination of interest rates, real output and inflation. Despite its simplicity, the model contains all the necessary ingredients for an insightful analysis of the stabilization effort of a central bank faced with a trade-off between inflation and the level of real activity. In particular, the private sector's forward looking behavior that naturally emerges from the model's microfoundations, and that sets it apart from its traditional Keynesian and "New Classical" predecessors, is at the heart of the time-inconsistency problem that opens the way to quasi-commitment.

After briefly describing the economic environment, in section (3.2) we derive an analytical solution to a version of the model with uncorrelated shocks. This helps to clarify the main steps involved in solving for a linear quasi commitment equilibrium and allows us to characterize some of its qualitative features. We then proceed to a quantitative investigation of the model's dynamic responses to the exogenous shocks and of their dependence on the credibility parameter $\alpha$. This naturally leads to the discussion of our main results on the distribution of the gains from commitment along the credibility dimension, which concludes the section.

\subsection{A Simple New Keynesian Model}

The demand side of the economy is described by a dynamic IS equation, which is simply a loglinear version of the Euler equation of the representative consumer

$$
x_{t}=E_{t} x_{t+1}-\sigma\left(i_{t}-E_{t} \pi_{t+1}-r_{t}^{n}\right)
$$

where $x_{t}$ is the output gap, $\pi_{t}$ is the rate of inflation, $i_{t}$ is the nominal interest rate controlled by the central bank and $r_{t}^{n}$ is the natural rate of interest, the real interest rate that would prevail in an equilibrium with flexible prices. The parameter $\sigma>0$ is the intertemporal elasticity of substitution in consumption. ${ }^{14}$

The supply behavior of the monopolistically competitive producers in the economy is described by a forward looking Phillips curve, obtained as a log-linear approximation to the optimal price setting rule under Calvo pricing

$$
\pi_{t}=\kappa x_{t}+\beta E_{t} \pi_{t+1}+u_{t}
$$

where $\beta$ is the subjective discount factor of the representative consumer, $\kappa>0$ is a function of structural parameters and $u_{t}$ is a "cost-push" shock (Clarida et al., 1999). As noted by Woodford (2003, Chapter 6), this is one of many supply shocks that might buffet this economy. It plays a crucial role here because of its inefficient nature. Driving a fluctuating wedge between the natural and the efficient level of output, this shock presents the monetary authority with a trade-off between output and inflation stabilization.

Finally, the period objective function of the monetary authority, derived as a second order approximation to the utility of the representative consumer, is of the usual quadratic form

$$
L_{t}=\pi_{t}^{2}+\lambda_{x} x_{t}^{2}
$$

\footnotetext{
${ }^{14}$ More precisely, since $i_{t}$ is interpreted here as the annualized value of the quarterly nominal interst rate, the intertemporal elasticity of substitution in quarterly consumption is $4 \sigma$.
} 
with the central bank's inflation and output gap targets both assumed to equal zero. This economy is thus characterized by the absence of the traditional average inflation bias of Barro and Gordon (1983). The steady state level of output is assumed to be efficient, for example because a sales subsidy eliminates the distortion associated with monopolistic competition, so that the central bank will not be tempted to push the economy above potential through surprise inflation. Nevertheless, as illustrated below, the commitment policy continues to deliver a better outcome than discretion, as it internalizes the beneficial effect of controlling expectations through promises about future policy behavior.

Note also that in the absence of cost-push shocks, the economy could be perfectly stabilized around the optimal values of zero inflation and output gap. It is only in response to an adverse shock say, that the central bank finds it optimal to engineer a recession. Under the optimal commitment plan, this recession will be protracted beyond what is strictly necessary to counteract the immediate inflationary effects of the shock. It is this extra dose of contraction, optimally promised at the beginning of the disinflation program to rein in inflation expectations, that makes the policy under commitment time inconsistent.

\subsection{Equilibrium Fluctuations under Quasi Commitment}

Having described the economic environment, we can now turn to the characterization of the equilibrium under quasi commitment. First, we derive analytical expressions for the equilibrium sequences of the endogenous variables $\left\{x_{t}, \pi_{t}, i_{t}\right\}_{t=\tau_{j}}^{\infty}$ within a regime, as a function of the credibility parameter $\alpha$, in an economy with i.i.d. shocks. We then investigate the dynamic behavior of a calibrated version of the model in response to both independent and autocorrelated inefficient shocks.

\subsubsection{Analytical Characterization}

The problem of maximizing the objective (15) under the constraints (13) and (14) can be greatly simplified if we note that a perfectly observable $r_{t}^{n}$ does not pose any particular problem to the policymaker, who can completely insulate the economy from fluctuations in the natural interest rate with offsetting movements in the policy instrument $i_{t}$. We can then proceed as if $x_{t}$ were the actual instrument of policy and the Phillips curve the only constraint for the policymaker, with the dynamic IS equation left to determine the level of the interest rate necessary to bring about the desired path for the output gap. The optimal policy problem can then be stated as

$$
\begin{array}{ll}
\min _{\left\{x_{t}\right\}_{t \geq 0}} & E_{0} \sum_{j=0}^{\infty} \beta^{\tau_{j}}\left[\sum_{k=0}^{\Delta \tau_{j}}\left(\pi_{\tau_{j}+k}^{2}+\lambda_{x} x_{\tau_{j}+k}^{2}\right)\right] \\
\text { s.t. } \quad 1_{\left\{\eta_{t+1}=0\right\}}\left[\beta E_{t} \pi_{t+1}-\pi_{t}+\kappa x_{t}+u_{t}=0\right] \\
\quad u_{0}=u
\end{array}
$$

where, as in equation (5), we have highlighted the losses pertaining to the $j^{\text {th }}$ central banker. As detailed above, the key to the solution of this problem is the correct treatment of expectations. 
The private sector survives its central bankers and therefore needs to systematically contemplate the possibility of a change in regime. In this simple model, in which the equilibrium inflation rate is assumed to be a linear function of the current cost-push shock, or $\pi_{\tau_{j}+k+1}=\pi^{*}+h u_{\tau_{j}+k+1}$, one step ahead expectations are formed as

$$
\begin{aligned}
E_{\tau_{j}+k} \pi_{\tau_{j}+k+1} & =(1-\alpha) E_{\tau_{j}+k}\left[\pi_{\tau_{j}+k+1} \mid \tau_{j+1}>\tau_{j}+k+1\right]+\alpha h\left(\pi^{*}+E_{\tau_{j}+k} u_{\tau_{j}+k+1}\right) \\
& =(1-\alpha) E_{\tau_{j}+k}\left[\pi_{\tau_{j}+k+1} \mid \tau_{j+1}>\tau_{j}+k+1\right]
\end{aligned}
$$

where the second line follows from the independence of $u_{t}$ and the fact that, with no average inflation bias, average inflation is zero (i.e. $\pi^{*}=0$ ). The Bellman equation associated with the $j^{\text {th }}$ central banker's problem (see equation (6) above) therefore becomes

$$
\begin{aligned}
V\left(u_{\tau_{j}}\right)= & \min _{\left\{x_{k}, \pi_{k}\right\}_{k \geq 0}} \max _{\left\{\varphi_{k+1}\right\}_{k \geq 0}} E_{\tau_{j}} \sum_{k=0}^{\infty}[\beta(1-\alpha)]^{k}\left\{\pi_{\tau_{j}+k}^{2}+\lambda_{x} x_{\tau_{j}+k}^{2}+\alpha \beta V\left(u_{\tau_{j}+k+1}\right)\right. \\
& \left.+2 \varphi_{\tau_{j}+k+1}\left[(1-\alpha) \beta E_{\tau_{j}+k}\left[\pi_{\tau_{j}+k+1} \mid \tau_{j+1}>\tau_{j}+k+1\right]-\pi_{\tau_{j}+k}+\kappa x_{\tau_{j}+k}+u_{\tau_{j}+k}\right]\right\}
\end{aligned}
$$

where $\varphi_{\tau_{j}+k+1}$, the Lagrange multiplier attached to the equation determining inflation at time $\tau_{j}+k$, is measurable with respect to the information set $\mathcal{I}_{\tau_{j}+k}$. Taking first order conditions of the extremum problem on the right hand side of (16) we obtain, $\forall t \in\left\{\tau_{j}, \ldots, \tau_{j}+\Delta \tau_{j}\right\}$

$$
\begin{aligned}
\lambda_{x} x_{t}+\kappa \varphi_{t+1} & =0 \\
\pi_{t}-\varphi_{t+1}+\varphi_{t} & =0 \\
(1-\alpha) \beta E_{t} \pi_{t+1} & =\pi_{t}-\kappa x_{t}-u_{t} \\
\varphi_{\tau_{j}} & =0
\end{aligned}
$$

This system, in which, with a slight abuse of notation, we have suppressed the proper conditioning in the one-step-ahead expectation in (17c), describes the evolution of the endogenous variables within the $j^{\text {th }}$ regime. Focusing our attention on equation (17a), we see that in the last period of the regime, $t_{L}=\tau_{j}+\Delta \tau_{j}$, the output gap can be written as $x_{t_{L}}=-\frac{\kappa}{\lambda_{x}} \varphi_{\tau_{j}+\Delta \tau_{j}+1}$. Given our definition of $\Delta \tau_{j} \equiv \tau_{j+1}-\tau_{j}-1$, this might seem to imply that $x_{t_{L}}=-\frac{\kappa}{\lambda_{x}} \varphi_{\tau_{j+1}}$, or that agents are aware of a coming change in regime before its realization. The solution to this apparent inconsistency is that $\tau_{j+1}$ is actually still a random variable from the perspective of time $t_{L}$. In other words, the public realizes that $t_{L}=\tau_{j}+\Delta \tau_{j}$ was indeed the last period of the $j^{\text {th }}$ regime only ex post, after having observed $\eta_{t_{L}+1}=1$. This is in turn reflected in the fact that, given our timing assumption on the multipliers, $\varphi_{\tau_{j}+\Delta \tau_{j}+1}$ is predetermined as of time $t_{L}$ (i.e. measurable with respect to $\mathcal{I}_{t_{L}}$ information), so that in fact $\varphi_{\tau_{j}+\Delta \tau_{j}+1} \neq \varphi_{\tau_{j+1}}=0$ and $x_{t_{L}}$ does not contain any information on the coming reoptimization. Moreover, again from equation (17a), we find that $\varphi_{t+1}=-\frac{\lambda_{x}}{\kappa} x_{t}$, which suggests that in this simple model the value of relaxing the forward looking constraint is proportional to the current output gap. This relationship therefore formalizes the intuition that the temptation to abandon the optimal plan is stronger, the deeper the recession to which the central bank has committed itself at the time of the shock. 
Now, combining the first two equations results in an equilibrium relationship between inflation and the output gap, $\pi_{t}=-\frac{\lambda_{x}}{\kappa} \Delta x_{t}$, which can in turn be substituted in (17c) to yield a second order difference equation for $x_{t}$

$$
(1-\alpha) \beta E_{t} x_{t+1}-\left((1-\alpha) \beta+\frac{\kappa^{2}}{\lambda_{x}}+1\right) x_{t}+x_{t-1}=\frac{\kappa}{\lambda_{x}} u_{t}
$$

with the "fictitious" initial condition $x_{\tau_{j}-1}=0$ (Woodford, 1999). Its characteristic polynomial

$$
(1-\alpha) \beta \mu^{2}-\left((1-\alpha) \beta+\frac{\kappa^{2}}{\lambda_{x}}+1\right) \mu+1=0
$$

has roots $\mu_{1}(\alpha)$ and $\mu_{2}(\alpha)$ inside and outside the unit circle respectively. Moreover, it can easily be shown that a marginal increase in credibility always decreases both roots, or, more formally, that $\frac{\partial \mu_{i}(\alpha)}{\partial \alpha}>0$, for $i=1,2$ and $\forall \alpha \in[0,1) .^{15}$

The solution of equation (18) is then

$$
x_{t}-\mu_{1} x_{t-1}=-\mu_{1} \frac{\kappa}{\lambda_{x}} u_{t}
$$

which, together with $x_{\tau_{j}-1}=0$, yields

$$
x_{\tau_{j}+k}=-\mu_{1} \frac{\kappa}{\lambda_{x}} \sum_{s=0}^{k} \mu_{1}^{s} u_{\tau_{j}+k-s}
$$

From this expression we can immediately observe that higher credibility (a lower $\alpha$ ), decreasing $\mu_{1}$, dampens the initial impact of a supply shock on the output gap and makes its decay faster. ${ }^{16}$ This in turn reflects the beneficial effect of credibility on inflation expectations, which translates into a favorable shift of the trade-off faced by the central bank. Another interesting feature of this equilibrium is that, by virtue of the quasi commitment assumption, each central banker's policy plan is entirely independent from the economic conditions prevailing before date $\tau_{j} .{ }^{17}$ This is reflected in the "initial" condition $x_{\tau_{j}-1}=0$, which is independent of the actual value of the output gap in period $\tau_{j}-1$. In other words, under quasi commitment, each central banker is assumed to contemplate her new plan from a $\tau_{j}$-optimal perspective, rather than from the timeless perspective of Woodford (1999) and Svensson and Woodford (2003). Under the timeless perspective, incoming central bankers would behave as if $x_{\tau_{j}-1}=x_{\tau_{j}-1}$, which would lead them to simply continue the plan initiated by their predecessor - who was indeed assumed to have done the same...- - thus eliminating the time inconsistency on which quasi commitment is built. It is important to note however that quasi commitment solves one of the logical inconsistencies of $t_{0^{-}}$ commitment, in that there is nothing special here about the times at which plans are reformulated,

\footnotetext{
${ }^{15}$ In the case of discretion $(\alpha=1)$ this solution method is no longer valid, since the infinite sum in (16) collapses to a single element and the first oder conditions in (17) no longer characterize the equilibrium.

16 This statement about the rate of decay is conditional on no reoptimization happening after the initial impulse, an event whose likelihood is inversely proportional to $\alpha$. See section (3.2.3) below for more details on this issue.

17 This is true only for the absence of "physical" state variables in our model (the $x$ 's of section 2 ). In the presence of such variables we would find instead that each central banker's plan is the same function of the initial state, a simple restatement of the Bellman principle holding across successive regimes.
} 
so that it becomes more acceptable that at those times policies would be reoptimized from a conditional rather than an unconditional perspective (Svensson, 1999b)

Now, iterating (17c) forward and making use of (19), we can also obtain an expression for the equilibrium inflation rate

$$
\pi_{t}=\frac{\kappa}{1-(1-\alpha) \beta \mu_{1}} x_{t}+u_{t}
$$

as a function of the output gap and of the current shock. Even if it is not possible to establish

analytically the effect of a marginal change in $\alpha$ on the equilibrium impact of an inefficient shock on inflation, this expression highlights the three sources of this credibility effect. First, fluctuations in inflation are inversely proportional to fluctuations in the (absolute value of the) output gap. Dampening the latter, credibility actually contributes to amplify the former, on impact. Second, accelerating the decay of the initial shock - conditional on no reoptimizations - more credibility leads the public to rationally forecast a faster easing of future inflationary pressures, thus contributing to moderate present inflation. This effect is reflected in the $-\beta \mu_{1}(\alpha)$ term in the denominator of (20). Third, a lower $\alpha$ means that agents, expecting any given regime to last longer, put more weight on their forecasts of future output gaps when translating them into their current pricing decisions, which in turn results in higher prices. This effect is reflected in the $-(1-\alpha)$ term in the denominator of $(20)$.

Finally, recalling the relationship between the current interest rate and the other endogenous variables given by (13), it is immediate to solve for the sequence of interest rates needed to bring about the equilibrium described above

$$
i_{t}=\left[\frac{\kappa \mu_{1}}{1-(1-\alpha) \beta \mu_{1}}-\sigma^{-1}\left(1-\mu_{1}\right)\right] x_{t}+r_{t}^{n}
$$

Since $i_{t}$ is a linear combination of the one step ahead forecasts of inflation and the output gap, it is obvious that if more credibility dampens the impact of shocks on both the equilibrium output gap and inflation, then it will have the same effect on the interest rate paths that implement that equilibrium. This intuition is confirmed by the preceding expression and by our numerical experiments below.

With these analytical expressions in hand, it is also possible to compute the value of the problem as a function of the credibility parameter $\alpha$. This expression though is too unwieldy to yield any particular insight and is therefore omitted. We choose instead to visually illustrate some of the features of the equilibrium resorting to a calibrated version of the model.

\subsubsection{Calibration}

Our benchmark calibration for the model's parameters follows Woodford (1999), and is summarized in the table below

\begin{tabular}{|c|c|c|c|}
\hline$\sigma$ & $\kappa$ & $\beta$ & $\lambda_{x}$ \\
\hline 1.5 & 0.1 & 0.99 & 0.048 \\
\hline
\end{tabular}

The model is assumed to refer to quarterly variables, with interest rates and inflation measured as annualized percentages. All assumed parameter values are reasonably standard, with the possible 
exception of the relative weight on the output gap in the central bank's loss function. This extremely low number derives from the microfoundations of the loss as a second order expansion of the utility function of the representative consumer. It is therefore consistent with the rest of the structural parameters. However, even if we adopt this value as our benchmark, we also report results for values of $\lambda_{x}$ more commonly found in the optimal monetary policy literature.

\subsubsection{Dynamic Responses to Inefficient Shocks}

Types of Impulse Response Functions The quasi commitment model presented in section ?? is driven by two different types of shocks: structural shocks $\varepsilon$ and regime change shocks $\eta$. When describing the dynamic behavior of the economy through impulse response functions, there are several possibilities that might reasonably be considered depending on what aspects of the model are under investigation.

Letting $z_{t}$ denote the variable under investigation, there are at least three types of impulse response functions (IRFs) one can plot in response to an impulse occurring at time $t=0$, for any given value of the credibility parameter $\alpha$. They are summarized in the table below

\begin{tabular}{|l|l|}
\hline & Impulse Response Function \\
\hline \hline i) & $E_{0}\left[z_{t} \mid \eta_{t}=0 \quad \forall t>0\right]-E_{-1}\left[z_{t}\right]$ \\
\hline ii) & $E_{0}\left[z_{t} \mid\right.$ random realization $\left.\left\{\eta_{t}\right\}_{t>0}\right]-E_{-1}\left[z_{t}\right]$ \\
\hline iii) & $E_{0}\left[z_{t}\right]-E_{-1}\left[z_{t}\right]$ \\
\hline
\end{tabular}

The first type of IRFs are what we call "within regime" responses. They are conditional on no reoptimization occurring over the horizon of interest and correspond to a traditional response from the state space form associated with the solution to system (17). Depending on the value of $\alpha$, the absence of a reoptimization becomes less and less likely with the length of the horizon, so that the path of $z_{t}$ is proportionally less representative of the actual position of the system. The second type of responses is one way to solve this problem. The path following a shock is now computed conditional on a sequence of reoptimizations (positive realizations of $\eta$ ), drawn from the distribution of the sequence $\left\{\eta_{t}\right\}$. These IRFs represent one possible realization of the path for the variable $z$, whose ex ante probability is indeed very low, but constant over the entire horizon. Finally, the responses under iii) are simply the ex ante average of all the possible conditional IRFs, integrated over the distribution of the corresponding reoptimization draws.

In the simple model above for example, noting that with i.i.d. shocks a reoptimization implies an immediate return to the steady state, analytical expressions for the IRFs of the output gap to an impulse $u_{0}$ can be written as

\begin{tabular}{|l|l|}
\hline \hline i) & $-\frac{\kappa}{\lambda_{x}} \mu_{1}^{t+1} u_{0}$ \\
\hline ii) & $-\frac{\kappa}{\lambda_{x}} \mu_{1}^{t+1} u_{0}$ if $\eta_{s}=0$ for $s=1, \ldots t$ \\
\hline iii) & $-\frac{\kappa}{\lambda_{x}} \mu_{1}\left[\mu_{1}(1-\alpha)\right]^{t} u_{0}$ \\
\hline
\end{tabular}


Impulse Responses to Independent Shocks With these preliminaries in place we can now turn to the description of the impulse responses of the simple monetary model presented in section 3.1 to a unit cost-push shock. ${ }^{18}$ We assume that the economy starts in steady state, with zero inflation and no output gap. Since these are also the values of the endogenous variables that maximize the policymaker's objective function, changes in regime will not move the economy away from this steady state as long as cost-push shocks are absent.

Figure 1 shows the path of inflation, output gap and interest rate under commitment and discretion. With the benchmark calibration, this just replicates the results in Woodford (1999). Under discretion the central bank moves its instrument with the shock, returning the economy to steady state as soon as the effects of the shock have faded. Given an i.i.d. impulse, this implies that the economy is driven into a sharp recession, accompanied by high inflation, but for only one period. Under commitment instead the central bank exploits the possibility of influencing inflation expectations in its favor in the period of the shock, by promising a protracted mild recession, accompanied by deflation. This can be accomplished with a very limited movement in the interest rate, in the absence of intervening shocks to the natural interest rate. This path for the policy variable is indeed time inconsistent, since the central bank would want to return to zero inflation and output gap as soon as the shock has disappeared. Note that this is also the policy that a new central banker would choose if allowed to reoptimize in any period following the period of the shock. In fact, in the absence of new shocks, the steady state values of the endogenous variables are consistent with an optimal commitment. ${ }^{19}$

What is the behavior of the economy under quasi commitment? Figure 2 provides part of the answer, presenting IRFs of type i) under two different levels of credibility, associated with an expected life of the commitment of two quarters and two years respectively. As noted above, this path for the variables is very unlikely, since it is associated with a string of twenty zero realizations of the $\eta$ shock following the cost-push impulse. Nevertheless this experiment is instructive. On the one hand, we see that the dynamic response of the system is almost indistinguishable from that under commitment when the average regime duration is $\alpha^{-1}=8$. This suggests that relatively low levels of credibility are enough to produce qualitative responses of the economy very close to the ones obtained under commitment. On the other hand, when the expected duration of the regime is only two quarters, the path of inflation is very close to that under commitment, but this is associated with a high cost in terms of output. The contraction needed to keep inflation close to its optimal level is much higher if the central bank has little credibility. This is the path that would obtain if, at the time of the shock, a new central banker came into office, who refused to validate the private sector's expectations and did not reoptimize even in the face of a positive realization of $\eta$. Of course, there is no room in our model for this sort of behavior. The optimal policy under quasi commitment entails a reoptimization whenever $\eta=1$, and the equilibrium is

\footnotetext{
${ }^{18}$ Impulses are normalized to produce an annualized one percentage point increase in inflation on impact, for given expectations. Given the forward looking nature of the model, the actual increase in inflation is a function of the forecasted response of policy to the shock.

19 This depends from the fact that there is no average inflation bias in our model. The inconsistency of the optimal plan is thus only conditional on the existence of inefficient shocks.
} 
constructed under the assumption that this will indeed happen. Nevertheless, we think that it is not hard to picture this as a crude description of what happened under Federal Reserve chairman Paul Volker. Inheriting a central bank with a low credibility in fighting inflation, faced with new inflationary pressures stemming from the second oil price shock, Volker chose to undergo the high output costs of a painful disinflation, ostensibly in order to establish a higher credibility for the Fed and thus better outcomes in the face of future shocks. To justify this behavior in a model where quasi commitment is the source of the lack of credibility, we should model a channel through which central bankers could convince the public that they are drawing the times of their reoptimizations from a distribution with a lower $\alpha$. This is one of the items on our research agenda.

To better characterize the "typical" behavior of the economy under quasi commitment, we need to take a stance on the occurrence of regime changes following a positive realization of the cost-push shock. This is done by looking at type ii) IRFs. The case with $\alpha^{-1}=2$ is depicted in Figure 3, where the circles mark the times of positive $\eta$ realizations. As expected, as soon as it is given the opportunity, the central bank moves the economy back to the optimal path under discretion, which, conditional on no new shocks hitting the economy, is also the optimal plan from the perspective of the time of the reoptimization. This is due to the absence of correlation in the inefficient shock. To see what happens on average, Figure 4 shows IRFs of type iii). The partially credible central bank generates intermediate dynamics between the discretion and commitment responses for the first few periods after impact, but that quickly converge to the discretion path after about four quarters.

Impulse Responses to Persistent Shocks Figure 5 shows what would happen if the system were hit by a persistent shock. ${ }^{20}$ In this case the difference in the path of the variables under discretion and commitment is less pronounced than before, since the persistence in the shock causes a similar persistence in inflation and output gap under discretion. In order to keep inflation close to its optimal path, a central banker that refused to reoptimize under quasi commitment would now cause a much sharper recession than under independent shocks, due to the prolonged impact of the shock on the economy and the associated higher inflation expectations. Somewhat stretching our interpretation, if we believe that the first oil price shock was expected to be persistent, while the second was thought to be temporary, this would lead us to conclude that Chairman Volker chose the optimal time to establish a higher credibility for the Fed. ${ }^{21}$ Finally, we can see how a series of reoptimizations, starting with one in period 3, now brings the endogenous variables back to their discretion paths only gradually. This is also confirmed by Figure 6, which shows impulse response functions of type iii). The convergence to the discretion path is much slower than in the uncorrelated case for inflation and the output gap, while the path of the policy instrument is virtually identical to that under discretion after only three quarters.

\footnotetext{
${ }^{20}$ We assume that $u_{t}$ is an $\mathrm{AR}(1)$ process with parameter 0.7 .

${ }^{21}$ It is interesting to note that the Fed did attempt to tighten policy in 1975, following the first oil price shock, but this attempt was quickly abandoned (a reoptimization?...). See for example De Long (1997) for a brief and suggestive monetary policy history of the sventies.
} 
Unconditional Moments A very similar picture to the one emerging from the conditional statements involved in studying impulse response functions also emerges from a look at the unconditional second moments of the equilibrium sequences. Figure 7 shows the unconditional correlations between the endogenous variables, for an economy with i.i.d. shocks. Once again we see how the ability to commit even for a short number of periods on average has dramatic effects on the equilibrium behavior of the economy, also contributing to a significant reduction in the volatility of the policy instrument that is required to achieve the desired equilibrium.

\subsection{The Gains from Commitment}

So far we have shown that our model's equilibrium dynamics are significantly influenced by policymakers' credibility, and that the continuum of quasi commitment rules bridges the gap between the commitment and discretion dynamics. Our next step is then to investigate to what extent credibility influences the level of welfare attainable under the optimal policy, conditional on the available commitment technology. Before doing this though, it is probably worth commenting on the metric that we will adopt for these welfare comparisons. If $y_{t}^{*}(\alpha)$ is the vector of equilibrium values for the arguments of the loss function, we can write the expected loss at the beginning of the planning horizon as $L(\alpha)=E\left[\sum_{t} u\left(y_{t}^{*}(\alpha)\right)\right]$. Although it is true that any monotonic transformation of $u$ maintains the same preference ordering, only affine transformations can be consistent with a given level of the elasticity of substitution. Therefore, for any given calibration, we can meaningfully interpret the change in welfare associated with different levels of credibility as a fraction of the total difference in welfare between discretion and commitment. This is the metric adopted below in our study of the gains from marginal increases in credibility.

Our analysis starts from Figure 8, which shows the effect on the lowest attainable loss of quarterly increments in the average length of a regime under our benchmark calibration. The gains from even minimal levels of credibility are very substantial and most of the total gain can be obtained with an expected duration of the commitment of about three years. More specifically, as shown in table 1, under the benchmark calibration an average regime length of six quarters is enough to produce $75 \%$ of the gains from commitment, and $90 \%$ of the gains can be obtained by an average duration of the commitment of three and a half years. This strong conclusion is robust to changes in the relative weight of output in the welfare function, as visually confirmed by Figure 10, in which inflation and output fluctuations receive the same wight. As shown in Table 1 , an average duration of the commitment of nineteen quarters is now required to obtain $75 \%$ of the gains from commitment, but two years are enough to cover half of the gap between discretion and commitment.

Another instructive way of looking at the significant effect of an even limited amount of commitment is to plot the combinations of output gap and inflation volatility associated with the optimal policy for different levels of $\alpha$. Figure 9 shows an even starker picture than the welfare comparisons. A commitment expected to last for only two quarters cuts the volatility of inflation by more than one half, with no discernible effect on the volatility of the output gap. An average 
duration of the commitment of one year is enough to move within two basis points of the inflation volatility under commitment, again with no significant losses in terms of output stabilization. Of course, further reductions in the volatility of inflation require significantly bigger sacrifices in terms of output volatility. In comparison, the volatility frontier in Figure 11 shows a much smoother transition from discretion to commitment in the case of $\lambda_{x}=1$, consistent with the corresponding results of the welfare ranking.

Finally, to illustrate the effect of credibility across all choices of output weights, Figure 12 displays the efficient frontiers traced out by letting $\lambda_{x}$ vary from our low benchmark value to infinity, with each line corresponding to a specific credibility level $\alpha$. Note that, as $\lambda_{x}$ increases towards infinity, all the curves converge to $(1,0)$ in $s d(\pi)-s d(x)$ space, since in this case, regardless of the level of $\alpha$, output is completely stabilized and the cost-push shocks feed directly into the inflation rate. What is interesting however is that, quite uniformly across output weights, we observe substantial welfare gains from marginal increases in credibility at low levels of expected regime duration.

\section{Conclusion}

This paper introduced the notion of quasi commitment, a policy rule with limited commitment in which policymakers renege on their announced optimal plans with a constant, exogenous probability every period. In this framework, commitment and discretion become the two polar cases of a more general decision making procedure. Assuming that private agents know the probability of a renegotiation, and form expectations on the future course of policy accordingly, we can interpret the expected duration of the announced plans as a continuous measure of whether policymakers match deeds to words. This is the notion of credibility proposed by Blinder (1998). We then apply our solution method to a calibrated version of a forward looking model of the monetary transmission mechanism. Our results confirm that, even in the absence of any reason for the central bank to induce a bias in the average level of inflation, commitment is superior to discretion in the natural metric of a second order approximation to the utility of the representative agent. More interestingly, we find that most of the gains from commitment accrue at relatively low levels of credibility. In our benchmark calibration, a commitment expected to last for six quarters is enough to bridge $75 \%$ of the welfare gap between discretion and commitment. We also show that this result is robust to variations in the welfare weight on output gap fluctuations.

This paper is only a preliminary study of the behavior of a particular model economy under quasi commitment. The robustness of our results across different models of the monetary transmission mechanism is an open question, that warrants further investigation. In fact, progress in this direction can be accomplished with a relatively modest effort, given that our solution method has been devised for a general class of DSGE models that admit a log-linear representation as an expectational VAR system.

Finally, endogenizing the probability of a change in regime seems an obvious step to increase the empirical plausibility of the model. We could think of two ways of accomplishing this, which 
we see as complementary. On the one hand, we could allow central bankers to invest in their credibility, for example by refusing to reoptimize when given the option. This would indeed be justified only as long as the private sector could learn about the new frequency of reoptimizations. Comparing the costs of the transition, when inflation expectations are higher than what is justified by the actual behavior of the central bank, with the long run gains associated with the higher level of credibility, would produce a normative analysis of the transition from low to high credibility regimes. On the other hand, and more in the spirit of Flood and Isard (1988), we could assume that the probability of a reoptimization depends on the state of the economy, for example by making this probability a function of the vector of predetermined Lagrange multipliers, which measure the temptation to disregard the associated constraints and reoptimize. Even though arguably desirable on the grounds of realism, these extensions would come at the cost of foregoing the linear quadratic structure that provides the model its extreme tractability. For this reason we think that the quasi commitment framework with exogenous reoptimizations provides a useful first step in the direction of expanding the menu of policy rules besides discretion and commitment. 


\section{A Some Details of the Calculations}

Below we provide a few additional details of the solution. A fully worked out set of calculations is available from the authors upon request.

\section{A.1 From the Bellman Equation to the Lagrangian}

The recursive formulation of the policymaker's problem stated regime by regime is given in (6) as

$$
\begin{gathered}
V\left(x_{\tau_{j}}\right)=\min _{\left\{x_{k}, X_{k}, i_{k}\right\}_{k=0}^{\infty}} \max _{\left\{\varphi_{k}\right\}_{k=0}^{\infty}} E_{\tau_{j}}\left\{\sum _ { k = 0 } ^ { \Delta \tau _ { j } } \beta ^ { k } \left[L_{\tau_{j}+k}+\beta^{\Delta \tau_{j}+1} V\left(x_{\tau_{j+1}}\right)\right.\right. \\
\left.\left.+2 \varphi_{\tau_{j}+k+1}^{\prime}\left(G E_{\tau_{j}+k} X_{\tau_{j}+k+1}-A_{21} x_{\tau_{j}+k}-A_{22} X_{\tau_{j}+k}-B_{2} i_{\tau_{j}+k}\right)\right]\right\} \\
\text { s.t. } \quad x_{\tau_{j}+k+1}-A_{11} x_{\tau_{j}+k}-A_{12} X_{\tau_{j}+k}-B_{1} i_{\tau_{j}+k}-\varepsilon_{\tau_{j}+k+1}=0 \\
\quad \varphi_{\tau_{j}}=0
\end{gathered}
$$

The policymaker calculates the first order condition of (21) taking as given private agents' rational expectation formation

$$
E_{\tau_{j+k}}\left[X_{\tau_{j}+k+1}\right]=(1-\alpha) E_{\tau_{j+k}}\left[X_{\tau_{j}+k+1} \mid \tau_{j+1}>\tau_{j}+k+1\right]+\alpha H E_{\tau_{j}+k}\left[x_{\tau_{j}+k+1}\right]
$$

as well as the form of the value function

$$
V(x)=x^{\prime} P x+\rho
$$

The running cost function in (21) involves a random sum since the policymaker is uncertain about how many periods the current regime will last. Thus she must take into account that the regime will last for any given number of periods with positive probability. In this context, the assumed exogeneity of the regime change signals represents a major simplification. In particular, the assumption implies that $\forall j: \Delta \tau_{j} \sim \operatorname{Geometric}(\alpha)$, so that $\operatorname{Pr}\{\Delta \tau=m\}=(1-\alpha)^{m} \alpha$.

Using the law of iterated expectations we can apply $E_{\tau_{j}+k}\left[\cdot \mid \tau_{j}=m\right]$ to the $k^{\text {th }}$ term in (21), where $k \leq m$. Note that

$$
\begin{aligned}
E_{\tau_{j}+k}\left[\varphi_{\tau_{j}+k+1}^{\prime} E_{\tau_{j}+k} X_{\tau_{j}+k+1} \mid \Delta \tau_{j}\right. & =m]=\varphi_{\tau_{j}+k+1}^{\prime} E_{\tau_{j}+k} X_{\tau_{j}+k+1} \\
E_{\tau_{j}}\left[\beta^{\Delta \tau+1} V\left(x_{\tau_{j+1}}\right) \mid \Delta \tau_{j}\right. & =m]=\beta^{m+1} E_{\tau_{j}} V\left(x_{\tau_{j}+m+1}\right)
\end{aligned}
$$

and that (22) and (23) may be substituted on the right hand side of (21). Finally, noting that

$$
E_{\tau_{j}}[\cdot]=\sum_{m=0}^{\infty} \alpha(1-\alpha)^{m} E_{\tau_{j}}\left[\cdot \mid \Delta \tau_{j}=m\right]
$$

and switching the sums over $k$ and $m$, one obtains the Lagrangian corresponding to (21) 


$$
\begin{aligned}
\mathcal{L}= & E_{\tau_{j}}\left\{\sum _ { m = 0 } ^ { \infty } ( \beta ( 1 - \alpha ) ) ^ { m } \left[L_{\tau_{j}+m}+\alpha \beta\left(x_{\tau_{j}+m+1}^{\prime} P x_{\tau_{j}+m+1}+\rho\right)\right.\right. \\
& +2 \varphi_{\tau_{j}+m+1}^{\prime}\left((1-\alpha) G E_{\tau_{j}+m}\left[X_{\tau_{j}+m+1} \mid \Delta \tau_{j}>m+1\right]\right. \\
& \left.+\alpha G H x_{\tau_{j}+m+1}-A_{21} x_{\tau_{j}+m}-A_{22} X_{\tau_{j}+m}-B_{2} i_{\tau_{j}+m}\right) \\
& \left.\left.+2 \phi_{\tau_{j}+m+1}^{\prime}\left(x_{\tau_{j}+1+m}-A_{11} x_{\tau_{j}+m}-A_{12} X_{\tau_{j}+m}-B_{1} i_{\tau_{j}+m}\right)\right]\right\}
\end{aligned}
$$

\section{A.2 Solving for the Value Function}

After solving the first order conditions of the Lagrangian (24), one may solve for the value function parameters $(P, \rho)$ by substituting the state space representation of the equilibrium dynamics (10) into the Bellman equation (6). Collecting quadratic terms in the state variables yields an expression for $P$

$$
P=\left(\begin{array}{c}
I_{n_{x} \times n_{x}} \\
0_{n_{X} \times n_{x}}
\end{array}\right)^{\prime} V_{1}\left(\begin{array}{c}
I_{n_{x} \times n_{x}} \\
0_{n_{X} \times n_{x}}
\end{array}\right)+\alpha \beta\left(\begin{array}{c}
I_{n_{x} \times n_{x}} \\
0_{n_{X} \times n_{x}}
\end{array}\right)^{\prime} M^{\prime} V_{2} M\left(\begin{array}{c}
I_{n_{x} \times n_{x}} \\
0_{n_{X} \times n_{x}}
\end{array}\right)
$$

where the matrices $V_{1}, V_{2}$ solve the Sylvester equations

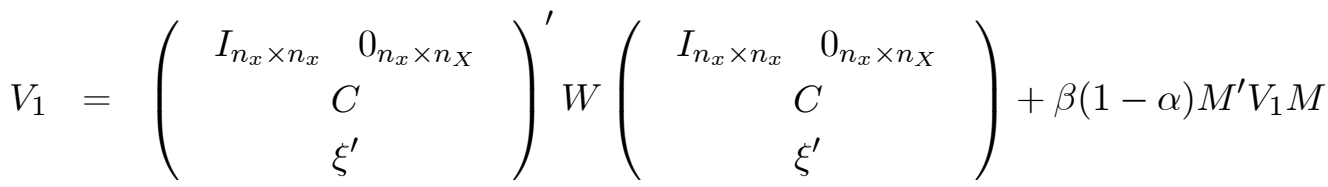

$$
\begin{aligned}
& V_{2}=\left(\begin{array}{c}
I_{n_{x} \times n_{x}} \\
0_{n_{X} \times n_{x}}
\end{array}\right) P\left(\begin{array}{c}
I_{n_{x} \times n_{x}} \\
0_{n_{X} \times n_{x}}
\end{array}\right)^{\prime}+\beta(1-\alpha) M^{\prime} V_{2} M
\end{aligned}
$$

Similarly, collecting constant terms yields an expression for $\rho$

$$
\begin{aligned}
\rho= & \frac{\beta}{1-\beta}\left((1-\alpha) \operatorname{tr}\left\{\left(\begin{array}{c}
I_{n_{x} \times n_{x}} \\
0_{n_{X} \times n_{x}}
\end{array}\right)^{\prime} V_{1}\left(\begin{array}{c}
I_{n_{x} \times n_{x}} \\
0_{n_{X} \times n_{x}}
\end{array}\right) \Omega\right\}\right) \\
& +\alpha \operatorname{tr}\left\{\left(\begin{array}{c}
I_{n_{x} \times n_{x}} \\
0_{n_{X} \times n_{x}}
\end{array}\right)^{\prime} V_{2}\left(\begin{array}{c}
I_{n_{x} \times n_{x}} \\
0_{n_{X} \times n_{x}}
\end{array}\right) \Omega\right\}
\end{aligned}
$$

Note that the matrices $V_{1}, V_{2}$ are functions of the state space form $\{M, C, \xi\}$ alone. Interestingly, one immediately retrieves the value function of the discretion problem by setting $\alpha=1$ (see for instance Söderlind, 1999). When $\alpha=0$ instead, the value function reduces to a Sylvester equation whose solution is the expected discounted sum of period losses conditional on the state space form holding forever. 


\section{References}

Abreu, Dilip, D. Pierce and E. Stacchetti (1986), "Optimal Cartel Equilibria with Imperfect Monitoring," Journal of Economic Theory 39:251-269.

Abreu, Dilip, D. Pierce and E. Stacchetti (1990), "Toward a Theory of Discounted Repeated Games with Imperfect Monitoring," Econometrica 58:1041-1063.

Barro, Robert J. and D.B. Gordon (1983), "A Positive Theory of Monetary Policy in a Natural Rate Model," Journal of Political Economy 91:589-610.

Barro, Robert J (1986), "Reputation in a Model of Monetary Policy with Incomplete Information" Journal of Monetary Economics 17: 3-20.

Blackburn, Keith and Michael Christensen (1989), "Monetary Policy and Policy Credibility: Theories and Evidence" Journal of Economic Literature 27: 1-45.

Blinder, Alan S. (1998), Central Banking in Theory and Practice, Cambridge, MA: MIT Press.

Calvo, Guillermo A. (1978), "On the Time Consistency of Optimal Policy in a Monetary Economy," Econometrica 46:1411-1428.

Kara, A. Hakan (2002), "Optimal Monetary Policy Rules under Imperfect Commitment," mimeo, New York University.

Chari V.V. and P. Kehoe (1990), "Sustainable Plans," Journal of Political Economy 98:783-802.

Clarida, Richard, Jordi Galí and Mark Gertler (1999), "The Science of Monetary Policy: A New Keynesian Perspective" Journal of Economic Literature 37: 1661-1707.

Cukierman, Alex (1992), Central Bank Strategy, Credibility, and Independence: Theory and Evidence, Cambridge: MIT Press.

Cukierman, Alex, Steven B. Webb and Bilin Neyapti (1992), "Measuring the Independence of Central Banks and Its Effect on Policy Outcomes" World Bank Economic Review 6:353-398.

DeLong, Bradford J. (1997), "America's Peacetime Inflation: The 1970s" in Christina Romer and David Romer (eds.) Reducing Inflation: Motivation and Strategy, Chicago: University of Chicago Press.

Faust, Jon and Lars E.O. Svensson (2000), "Transparency and Credibility: Monetary Policy with Unobservable Goals," International Economic Review 42:369-397.

Fischer, Stanley (1980), "Dynamic Inconsistency, Cooperation and the Benevolent Dissembling Government," Journal of Economic Dynamics and Control 2:93-107.

Flood and Isard (1988), "Monetary Policy Strategies," NBER Working Paper No. 2770.

Galí, Jordi (2001), "New Perspectives on Monetary Policy, Inflation, and the Business Cycle", in M. Dewatripont, L. Hansen and S. Turnovsky (eds.) Advances in Economics and Econometrics, the Eight World Congress, Cambridge, UK: Cambridge University Press. 
Kydland, Finn E. and Edward C. Prescott (1977), "Rules Rather than Discretion: The Inconsistency of Optimal Plans" Journal of Political Economy 85: 473-491.

Ljungquist, Lars and T. Sargent (2000), Recursive Macroeconomics Theory, Boston: MIT Press Marcet, Albert and Ramon Marimon (1999), "Recursive Contracts" mimeo, Universitat Pompeu Fabra

McCallum, Bennett T. (1999), "Issues in the Design of Monetary Policy Rules" in J.B. Taylor and M. Woodford (eds.) Handbook of Macroeconomics, Amsterdam: North-Holland.

Phelan, Christopher (2001), "Public Trust and Government Betrayal," mimeo, Federal Reserve Bank of Minneapolis.

Phelan, Christopher and E. Stacchetti (2001), "Sequential Equilibria in a Ramsey Tax Model," Econometrica 69:1491-1518.

Roberds, William (1987), "Models of Policy Under Stochastic Replanning," International Economic Review, 28:731-755.

Rogoff, Kenneth (1985), "The Optimal Degree of Commitment to a Monetary Target," Quarterly Journal of Economics 100:1169-1190

Rogoff (1989) "Reputation, Coordination and Monetary Policy" in Robert J. Barro (ed.) Modern Business Cycle Theory, Cambridge, MA: Harvard University Press.

Söderlind, Paul (1999), "Solution and Estimation of RE Macromodels with Optimal Policy," European Economic Review 41:111-1146.

Stokey, Nancy (1989), "Reputation and Time Consistency," American Economic Review 79:134139.

Stokey, Nancy (1991), "Credible Public Policy," Journal of Economic Dynamics and Control 15:627-657.

Svensson, Lars E.O. (1999a), "Inflation Targeting as a Monetary Policy Rule", Journal of Monetary Economics 43: 607-654.

Svensson, Lars E. O. (1999b), "How Should Monetary Policy Be Conducted in an Era of Price Stability?" in New Challenges for Monetary Policy, a symposium sponsored by the Federal Reserve Bank of Kansas City.

Svensson, Lars E.O.and Michael D. Woodford (2003), "Implementing Optimal Policy through Inflation-Forecast Targeting," in Bernanke, Ben S. and Michael Woodford (eds.) Inflation Targeting, Chicago: University of Chicago Press.

Woodford, Michael D. (1999), "Commentary: How Should Monetary Policy Be Conducted in an Era of Price Stability?"in New Challenges for Monetary Policy, a symposium sponsored by the Federal Reserve Bank of Kansas City.

Woodford, Michael D. (2003), Interest and Prices: Foundations of a Theory of Monetary Policy, Princeton: Princeton University Press. 


\section{Figures and Tables}
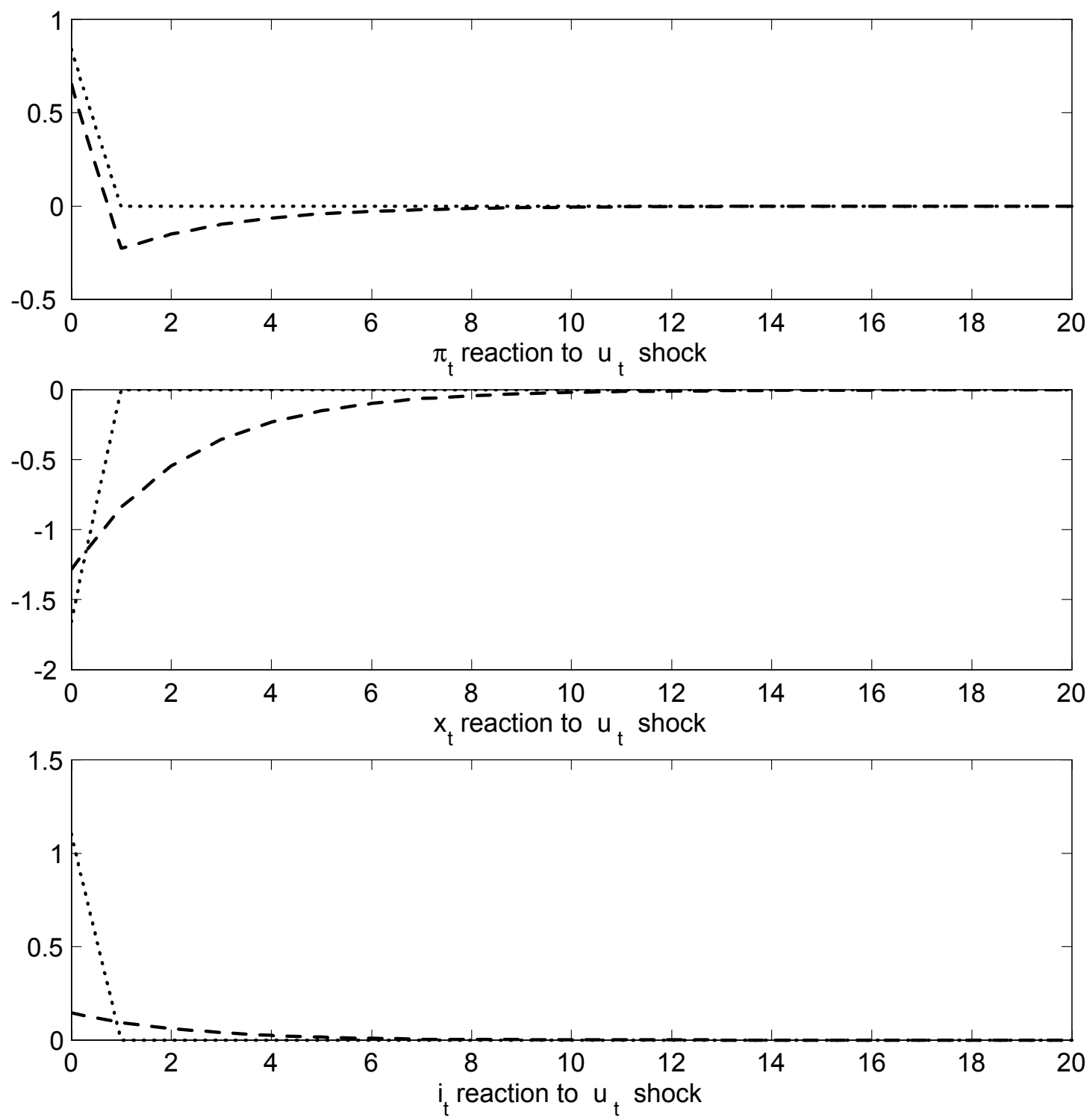

Figure 1: Dynamic responses to a one standard deviation, uncorrelated cost push shock under commitment and discretion. The fat dotted lines are the discretion responses and the fat dashed lines are the commitment responses. The weight on output in the loss function is $\lambda_{x}=0.05$. 

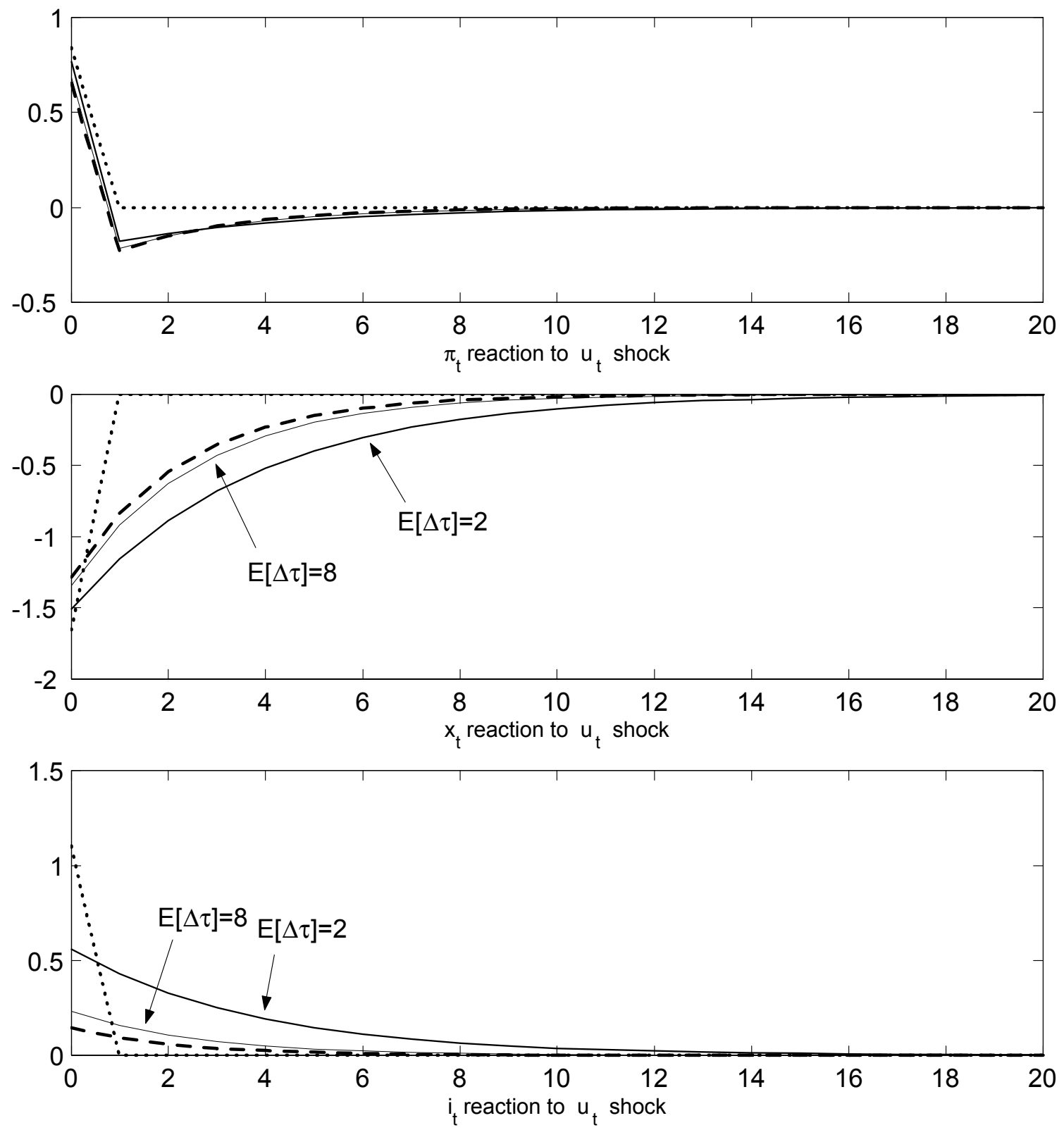

Figure 2: Dynamic responses to a one standard deviation, uncorrelated cost push shock with NO REOPTIMIZATIONS. The fat dotted lines are the discretion responses, the fat dashed lines are the commitment responses while the thin lines are the quasi commitment responses corresponding to average regime durations of 2 and 8 periods respectively (i.e. $\alpha=0.5,0.125$ ). The weight on output in the loss function is $\lambda_{x}=0.05$. 

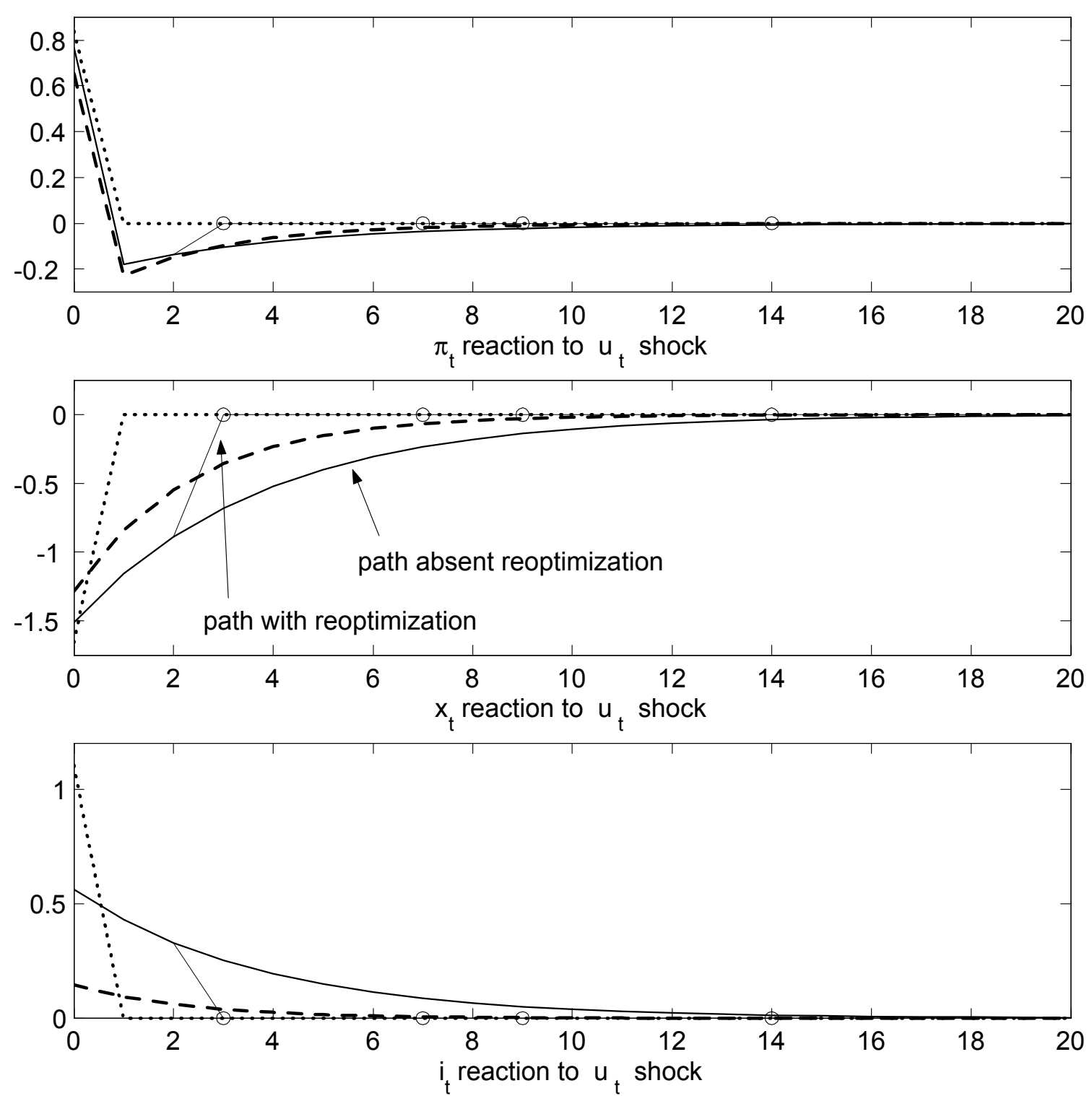

Figure 3: Dynamic responses to a one standard deviation, uncorrelated cost push shock with RANDOM REOPTIMIZATIONS. The fat dotted lines are the discretion responses, the fat dashed lines are the commitment responses while the thin lines are the quasi commitment responses corresponding to an average regime duration of 2 periods (i.e. $\alpha=0.5$ ). The circles correspond to periods in which reoptimization occurs. The weight on output in the loss function is $\lambda_{x}=0.05$. 

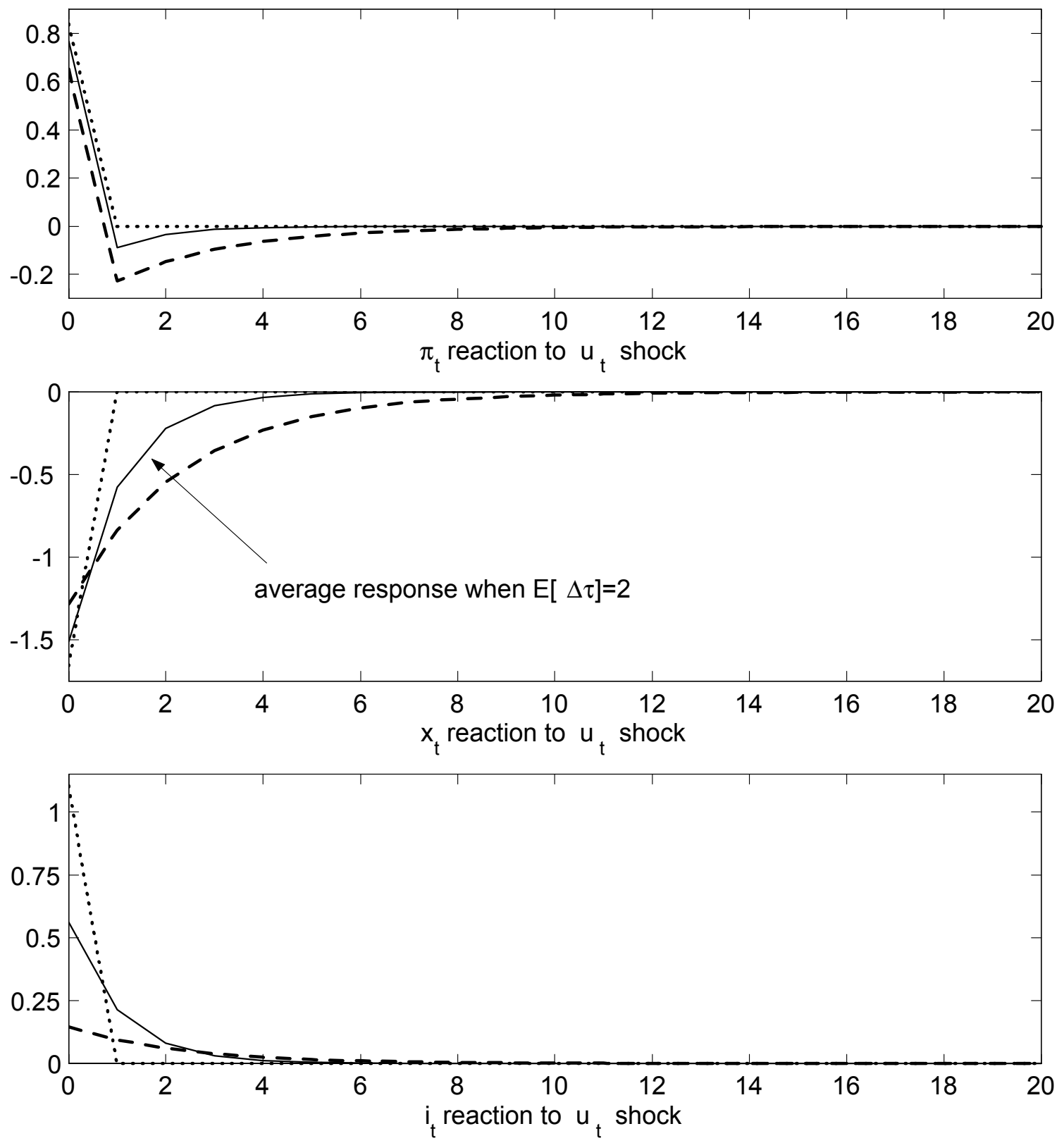

Figure 4: Dynamic responses to a one standard deviation, uncorrelated cost push shock, AVERAGING OVER REGIME CHANGES. The fat dotted lines are the discretion responses, the fat dashed lines are the commitment responses while the continuous lines are the average quasi commitment responses corresponding to an average regime duration of 2 periods (i.e. $\alpha=0.5$ ). The weight on output in the loss function is $\lambda_{x}=0.05$. 

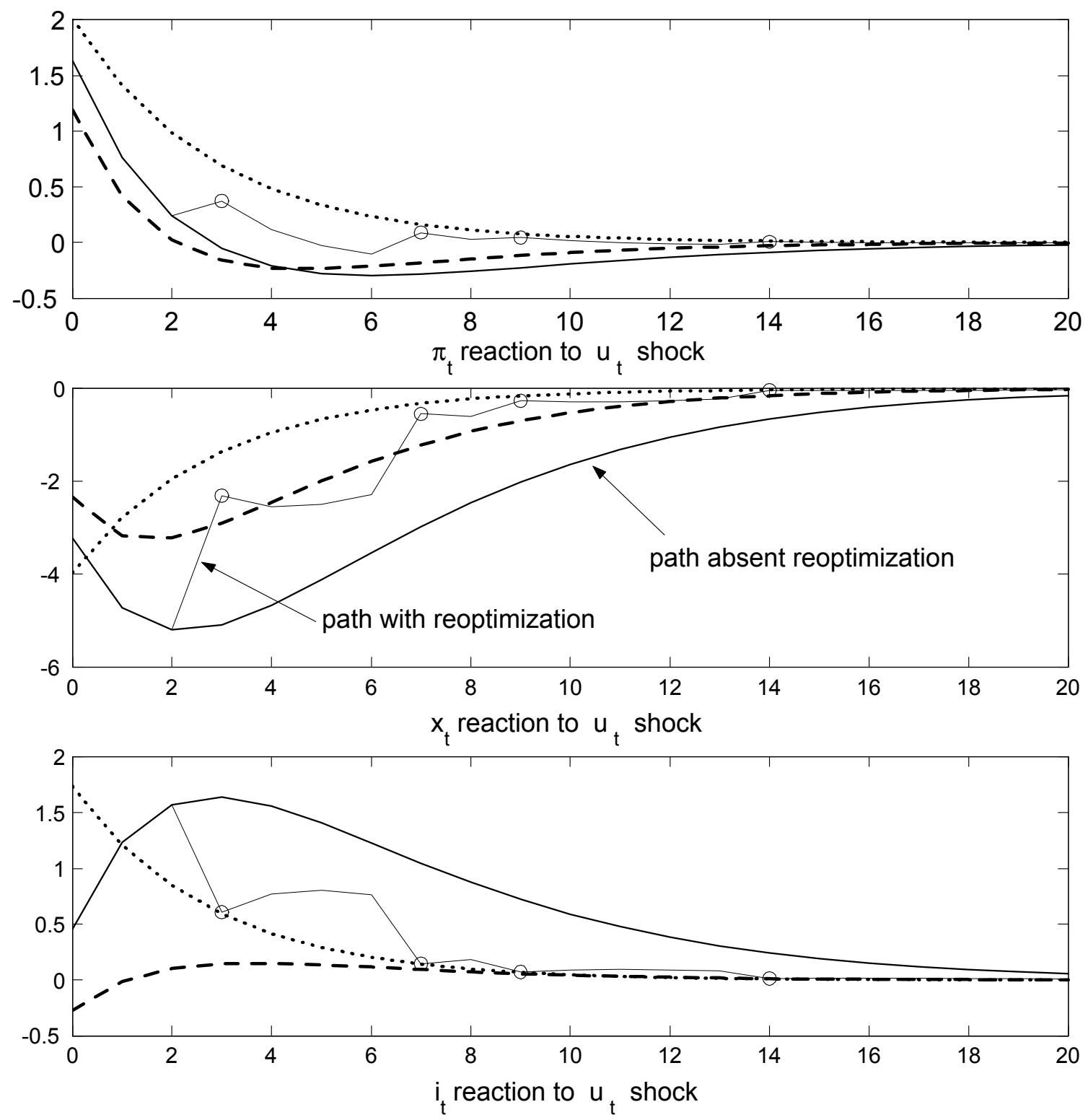

Figure 5: Dynamic responses to a one standard deviation, serially correlated $\left(\rho_{u}=0.7\right)$ cost push shock with RANDOM REOPTIMIZATIONS. The fat dotted lines are the discretion responses, the fat dashed lines are the commitment responses while the thin lines are the quasi commitment responses corresponding to an average regime duration of 2 periods (i.e. $\alpha=0.5$ ). The circles correspond to periods in which reoptimization occurs. The weight on output in the loss function is $\lambda_{x}=0.05$. 

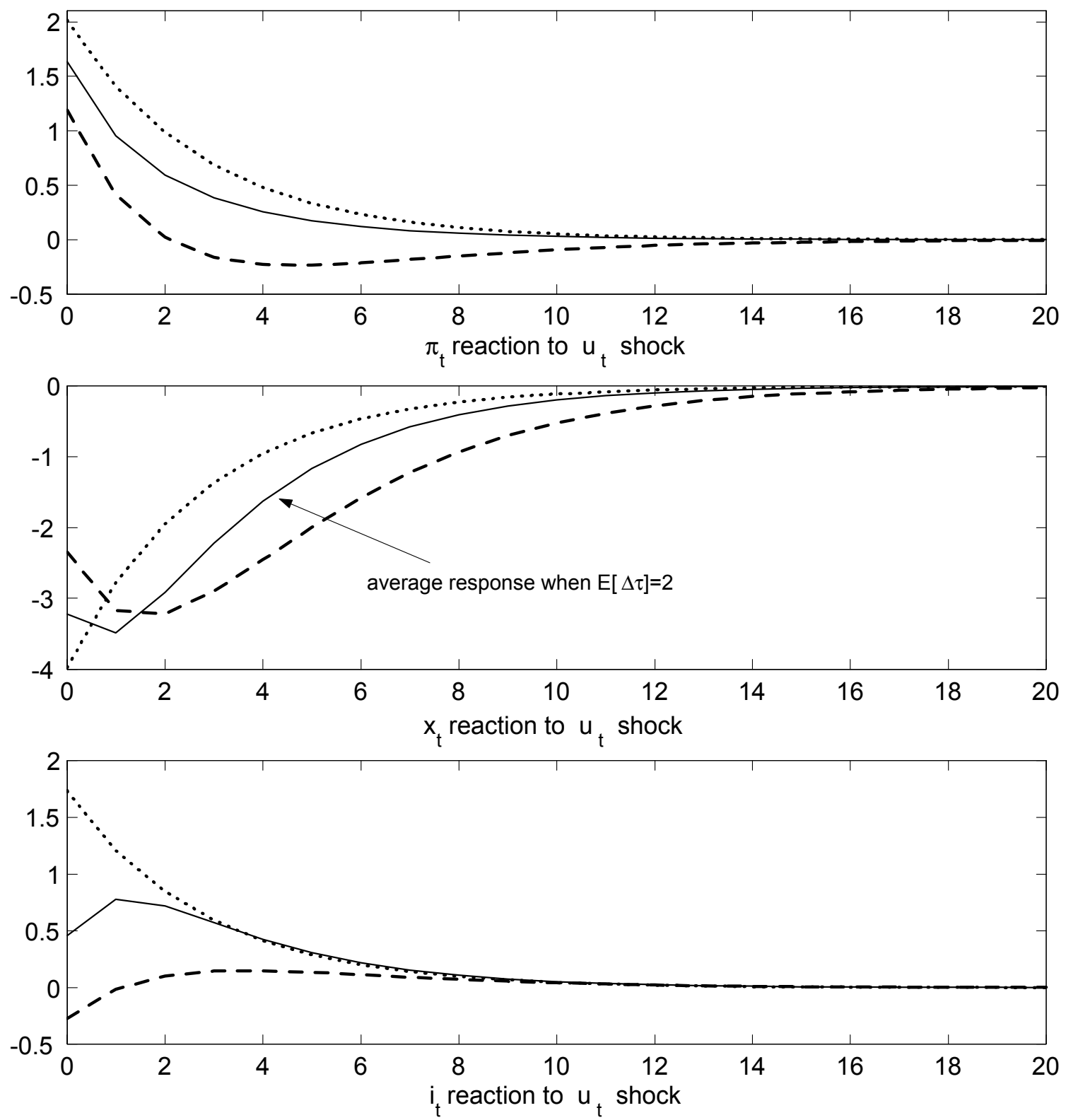

Figure 6: Dynamic responses to a one standard deviation, serially correlated $\left(\rho_{u}=0.7\right)$ cost push shock, AVERAGING OVER REGIME CHANGES. The fat dotted lines are the discretion responses, the fat dashed lines are the commitment responses while the continuous lines are the average quasi commitment responses corresponding to an average regime duration of 2 periods (i.e. $\alpha=0.125$ ). The weight on output in the loss function is $\lambda_{x}=0.05$. 

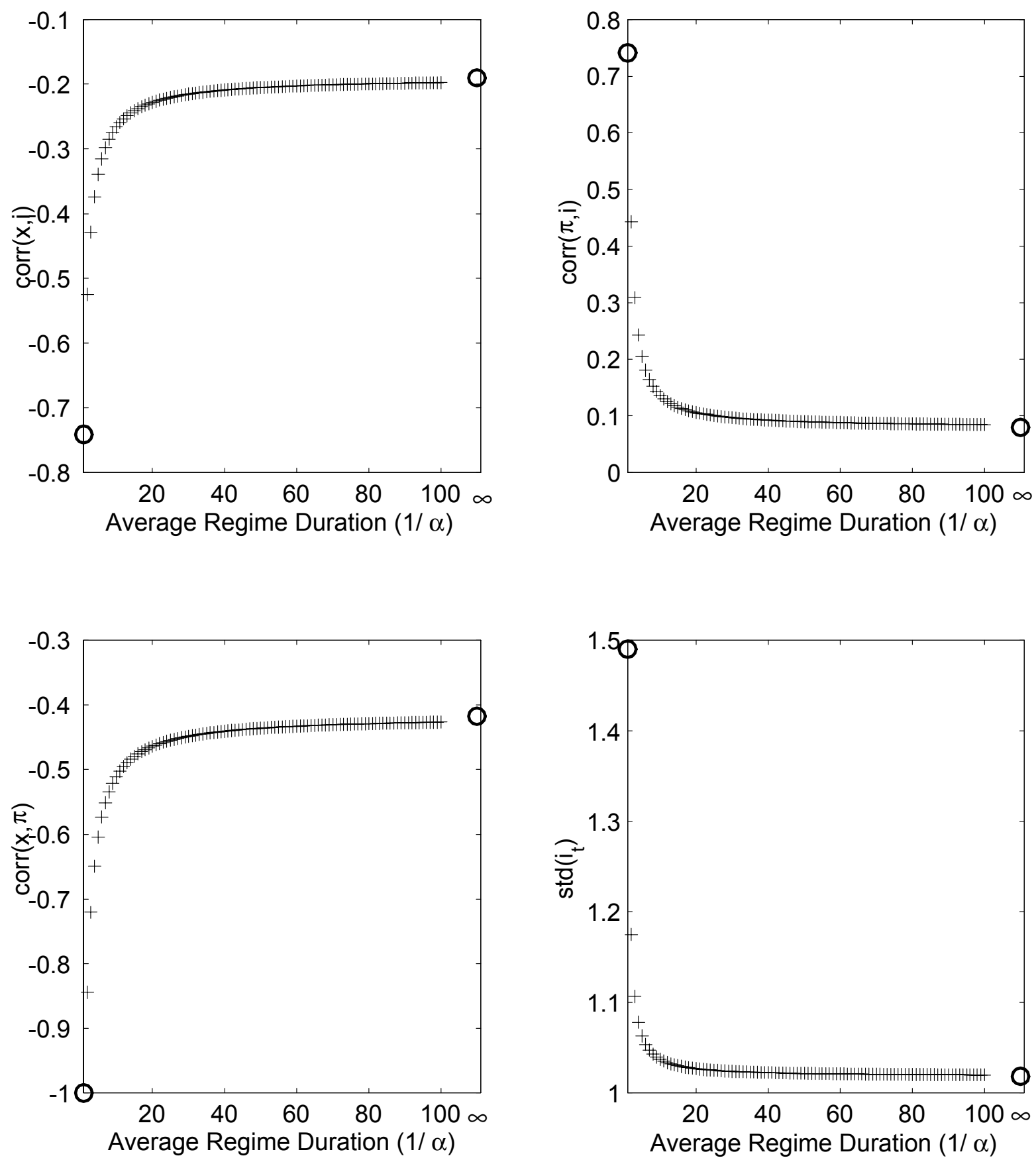

Figure 7: The equilibrium behavior of variables under various degrees of commitment. Each "+" corresponds to an average regime duration (in quarters). The discretion and limiting commitment cases are marked by circles. The weight on output in the loss function is $\lambda_{x}=0.05$ and the cost push shocks $u_{t}$ are uncorrelated. 


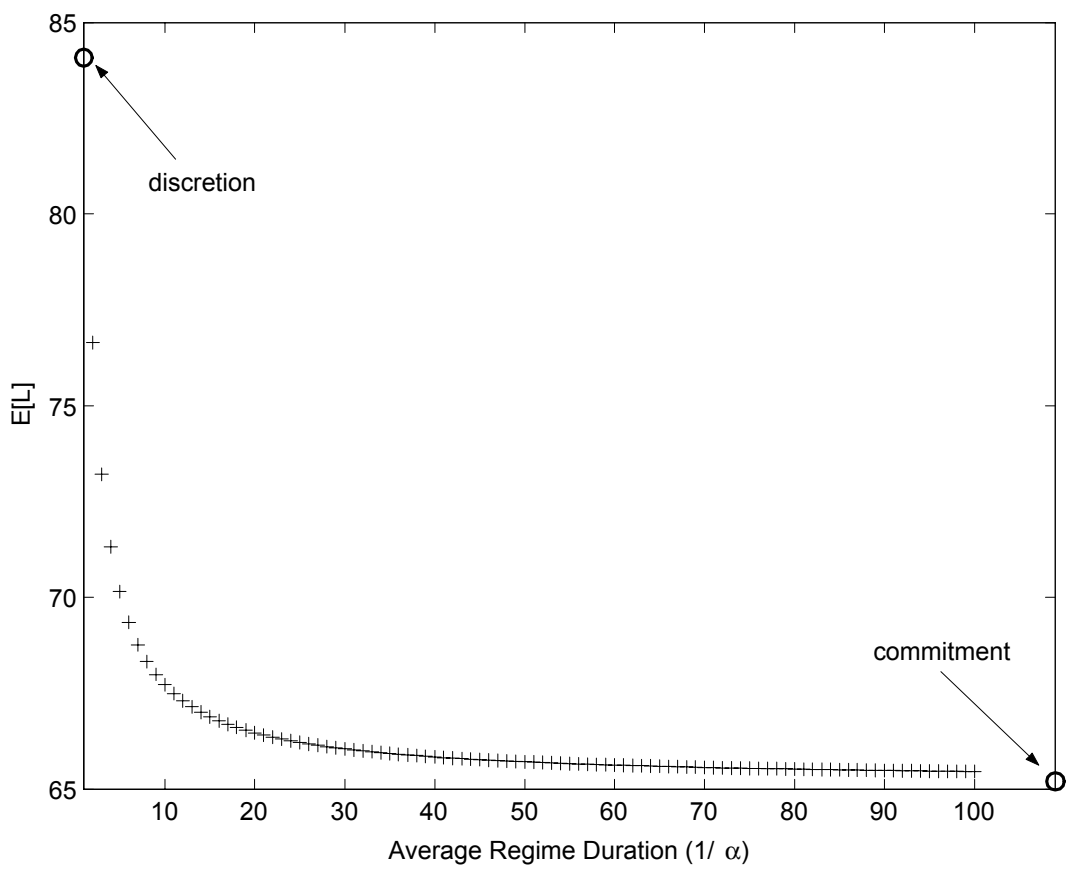

Figure 8: The gains from credibility. Each "+" corresponds to an average regime duration (in quarters). The limiting cases of discretion and commitment are marked by circles. The weight on output is $\lambda_{x}=0.05$ and the cost push shocks $u_{t}$ are uncorrelated.

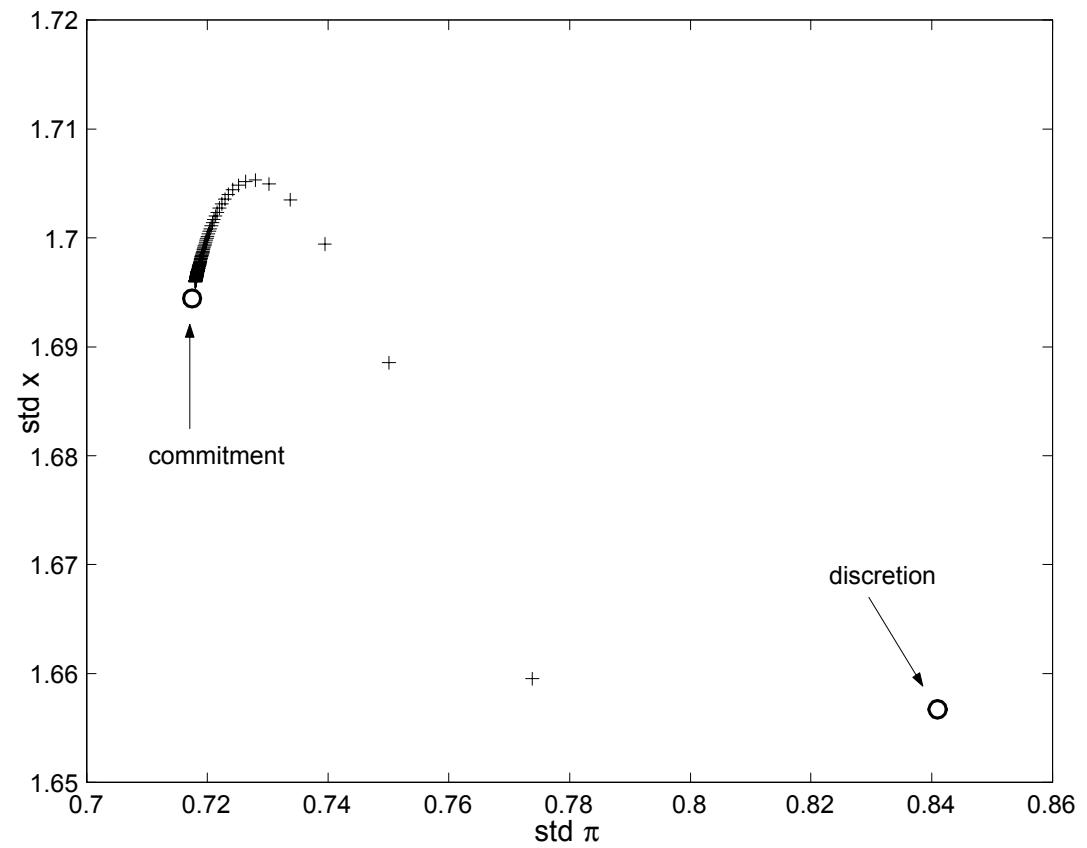

Figure 9: The efficient frontier as a function of the average credibility. Each "+" corresponds to an average regime duration (in quarters). The limiting cases of discretion and commitment are marked by circles. The weight on output is $\lambda_{x}=0.05$ and the cost push shocks $u_{t}$ are uncorrelated. 


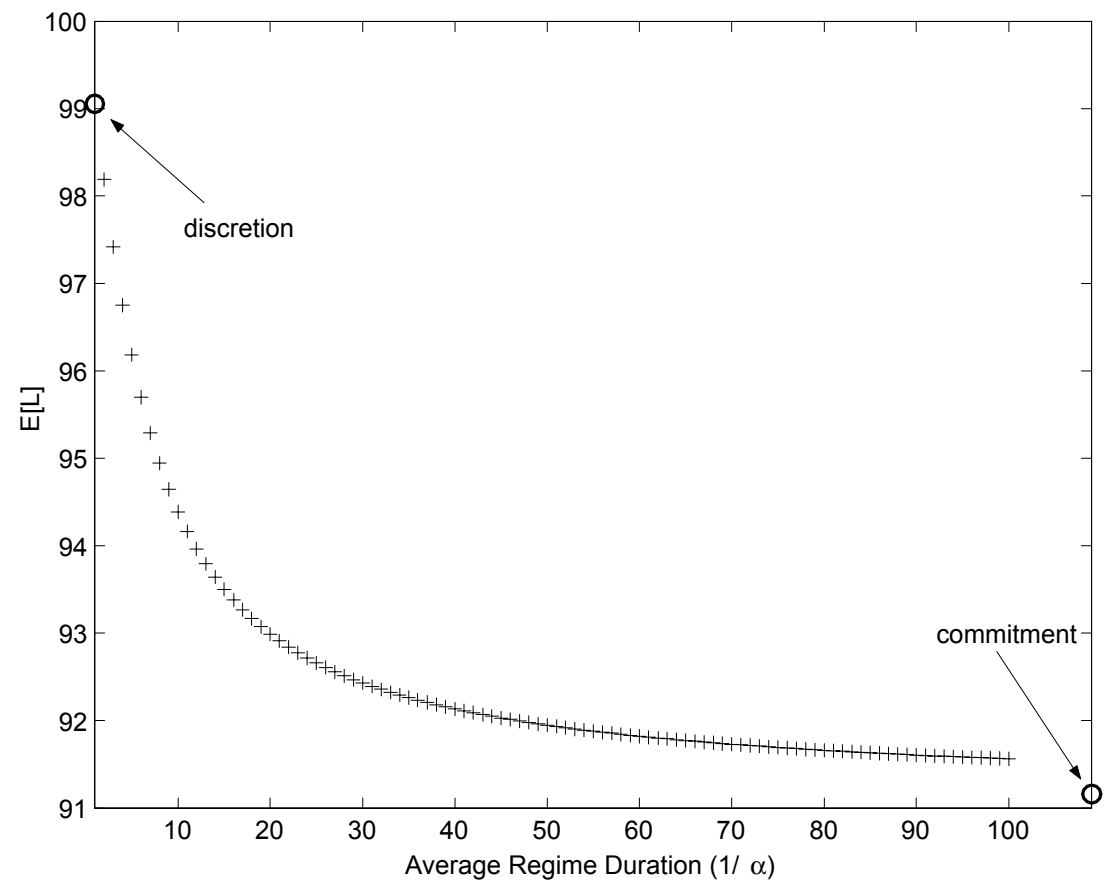

Figure 10: The gains from credibility. Each "+" corresponds to an average regime duration (in quarters). The limiting cases of discretion and commitment are marked by circles. The weight on output is $\lambda_{x}=1$ and the cost push shocks $u_{t}$ are uncorrelated..

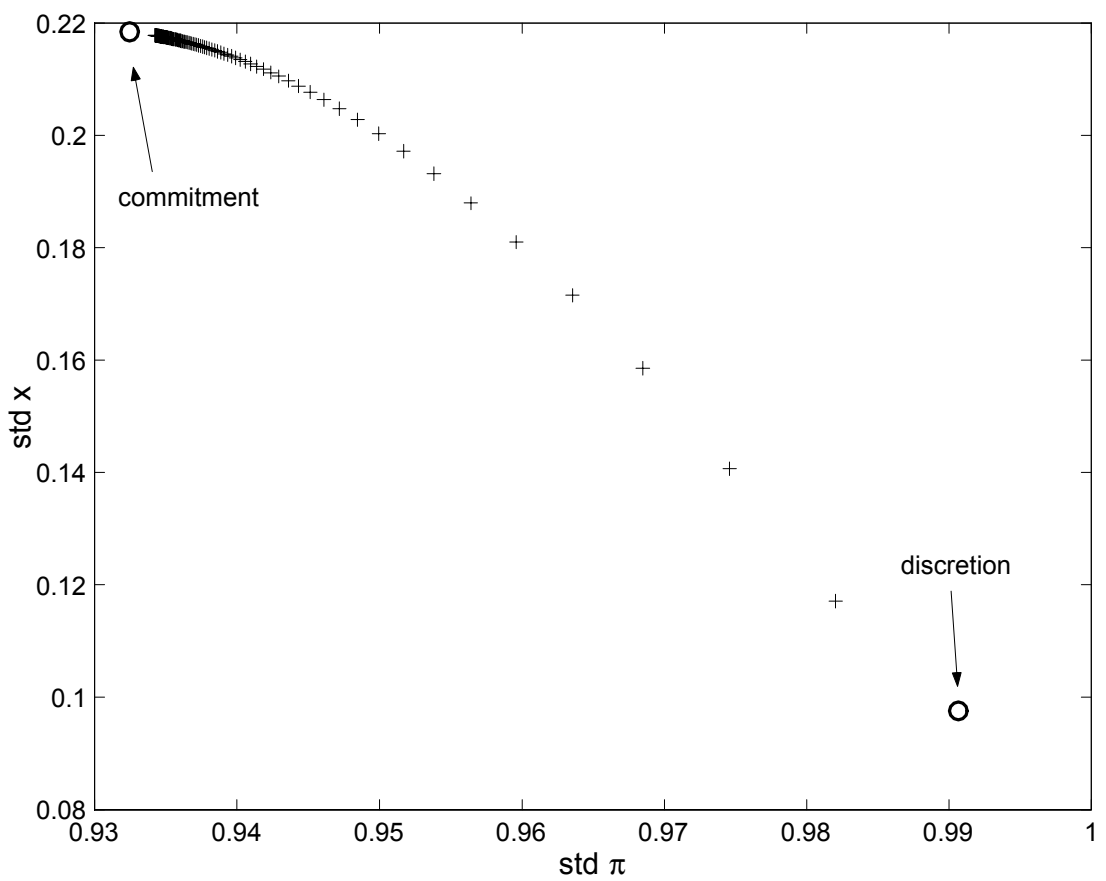

Figure 11: The efficient frontier as a function of the average credibility. Each "+" corresponds to an average regime duration (in quarters). The limiting cases of discretion and commitment are marked by circles. The weight on output is $\lambda_{x}=1$ and the cost push shocks $u_{t}$ are uncorrelated. 


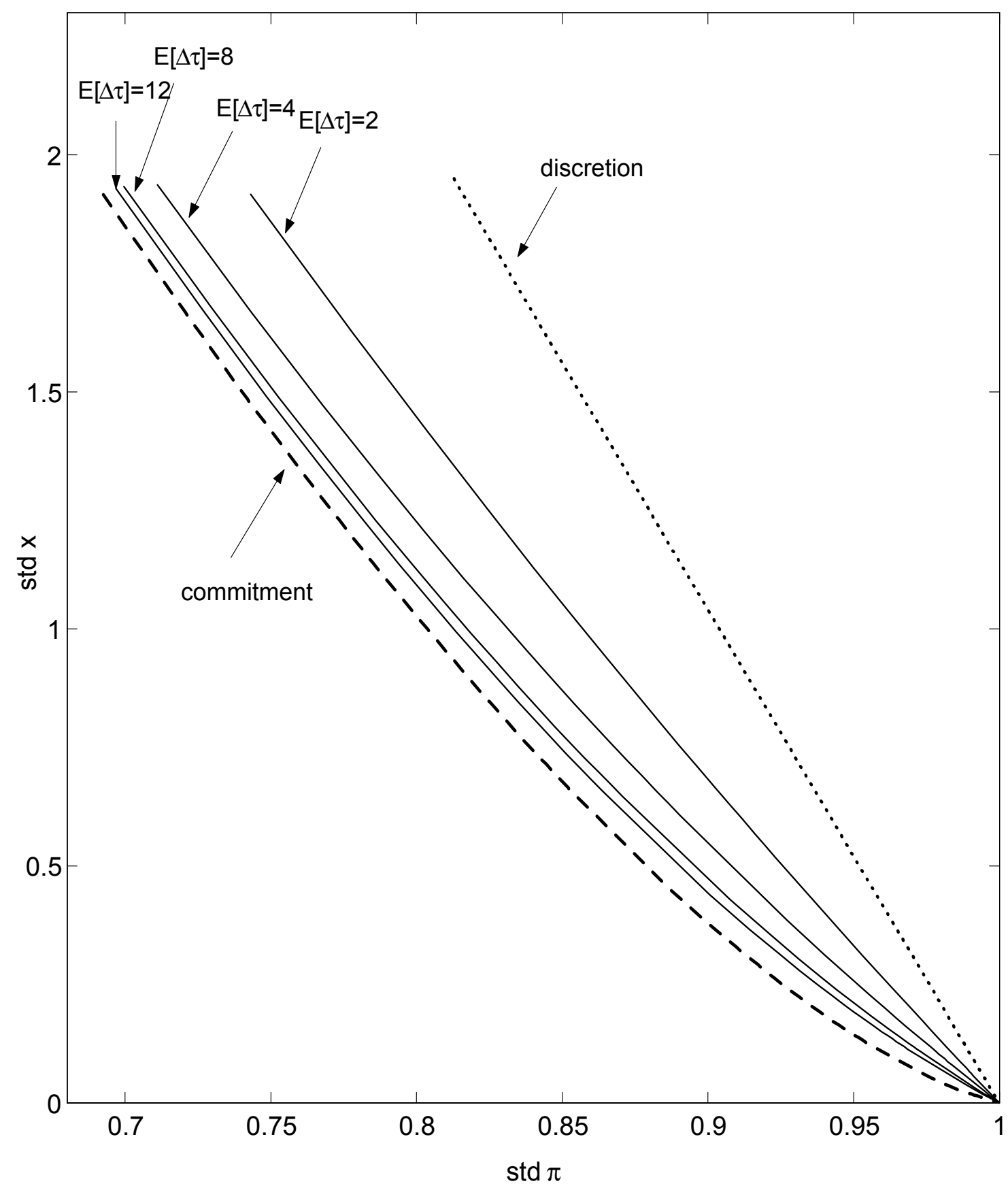

Figure 12: The efficient frontier as a function of the weight on output in the loss function, $\lambda_{x} \in[0.04 ; \infty)$. Each line corresponds to a different average regime duration (and thus a different $\alpha$ ). The leftmost line corresponds to commitment while the rightmost line corresponds to discretion. The lines in between correspond to (from right to left) average regime durations of $2,4,8$ and 12 quarters. 


\begin{tabular}{cc|cccc} 
& & \multicolumn{4}{c}{ Weight on Output: $\lambda_{x}$} \\
& & 0.05 & 0.25 & 0.50 & 1.00 \\
\cline { 3 - 6 } $\mathrm{G}$ & $25 \%$ & 2 & 3 & 3 & 4 \\
$\mathrm{a}$ & $(39 \%)$ & $(37 \%)$ & $(28 \%)$ & $(29 \%)$ \\
$\mathrm{a}$ & $50 \%$ & 3 & 5 & 6 & 8 \\
$\mathrm{i}$ & & $(57 \%)$ & $(56 \%)$ & $(52 \%)$ & $(52 \%)$ \\
$\mathrm{n}$ & $75 \%$ & 6 & 10 & 14 & 19 \\
& & $(78 \%)$ & $(76 \%)$ & $(76 \%)$ & $(76 \%)$ \\
& $90 \%$ & 14 & 26 & 36 & 50 \\
& & $(90 \%)$ & $(90 \%)$ & $(90 \%)$ & $(90 \%)$
\end{tabular}

Table 1: The table entries show how many periods the central bank needs to commit for, on average, in order to achive at least some given percentage of the total gain from commitment. The numbers in paranthesis are the actual percentage gains. They differ because only integer (average) commitment lengths are considered. 